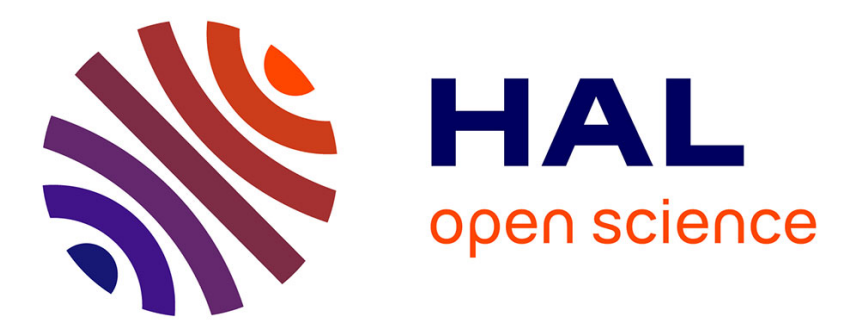

\title{
Phénomènes de relaxation et frottement intérieur dans les solides vitreux
}

\author{
J. Perez, P.F. Gobin
}

\section{To cite this version:}

J. Perez, P.F. Gobin. Phénomènes de relaxation et frottement intérieur dans les solides vitreux. Revue de Physique Appliquée, 1977, 12 (5), pp.819-836. 10.1051/rphysap:01977001205081900 . jpa00244248

\section{HAL Id: jpa-00244248 https://hal.science/jpa-00244248}

Submitted on 1 Jan 1977

HAL is a multi-disciplinary open access archive for the deposit and dissemination of scientific research documents, whether they are published or not. The documents may come from teaching and research institutions in France or abroad, or from public or private research centers.
L'archive ouverte pluridisciplinaire HAL, est destinée au dépôt et à la diffusion de documents scientifiques de niveau recherche, publiés ou non, émanant des établissements d'enseignement et de recherche français ou étrangers, des laboratoires publics ou privés. 


\title{
PHÉNOMĒNES DE RELAXATION ET FROTTEMENT INTÉRIEUR DANS LES SOLIDES VITREUX
}

\author{
J. PEREZ et P. F. GOBIN
}

Groupe d'Etudes de Métallurgie Physique et de Physique des Matériaux E. R. A. no 463, I. N. S. A. Villeurbanne 69621, France

Résumé. - Les solides vitreux présentent divers phénomènes de relaxation mécanique que l'on peut étudier par mesure de frottement intérieur. Les caractéristiques de ces phénomènes varient d'un type de solides vitreux à un autre. Néanmoins, on peut relever certains comportements qui semblent communs à l'ensemble des solides à l'état vitreux. Ainsi, le rappel de résultats concernant :

- les verres minéraux,

- les matériaux organiques vitreux,

- les solides covalents vitreux,

- les verres métalliques,

fait apparaître que, dans tous les cas, il existe un processus de relaxation vers le domaine de transition vitreuse (relaxation primaire) et des processus de relaxation plus ou moins bien différenciés à plus basse température (relaxation secondaire).

La relaxation primaire ou $\alpha$ qui se traduit généralement par une montée rapide du frottement intérieur avec la température est caractérisée par la non-validité de la loi d'Arrhénius et la nonlinéarité vis-à-vis de la contrainte.

Les phénomènes de relaxation secondaire, au contraire, sont linéaires et suivent la loi d'Arrhénius. Ils peuvent être nombreux et si certains peuvent être expliqués en envisageant le mouvement d'éléments structuraux bien définis (par exemple, saut des ions alcalins dans un verre silice-oxyde alcalin), d'autres donnent lieu à des anomalies de frottement intérieur dont l'interprétation n'est pas toujours clairement établie. Ainsi, un peu au-dessous du domaine de transition vitreuse, on observe souvent un pic ou une anomalie de frottement intérieur plus ou moins bien résolue de la montée de frottement intérieur lié à la relaxation $\alpha$; cette manifestation semble être associée à des effets d'élasticité retardée. Un tel comportement nous paraît correspondre à un $2^{\mathrm{e}}$ phénomène de relaxation propre à l'état vitreux (relaxation $\beta$ ).

Pour concilier les données concernant les modèles structuraux proposés pour décrire l'état vitreux et la nécessité d'envisager 2 environnements atomiques différents pour rendre compte des phénomènes de relaxation $\alpha$ et $\beta$, nous proposons d'ajouter aux modèles de structure généralement admis l'hypothèse suivant laquelle existeraient des défauts de type interfaces. La relaxation $\beta$ pourrait ainsi résulter du mouvement des atomes ou molécules au niveau des interfaces alors que la relaxation $\alpha$ serait la conséquence du mouvement coopératif des atomes ou des molécules entre les interfaces.

Dans une première approche, l'adaptation des modèles de relaxation déjà connus dans le cas des solides cristallins aux solides vitreux nous permet de rendre compte, au moins qualitativement, des résultats expérimentaux.

Abstract. - Several mechanical relaxation phenomena are observed on glasses. The characteristics of these phenomena varry from one type of glass to another but some features seem to be common to all solids in the vitreous state. Hence, results about :

- oxyde glasses,

- organic glasses,

- vitreous semiconductors,

- metallic glasses,

are recalled. Then, it is shown that there are a relaxation process about the glass transition (primary relaxation) and several relaxation processes more or less differenciated at lower temperature (secondary relaxations).

The primary or $\alpha$ relaxation corresponds to a rapid increase of internal friction with temperature ; the Arrhenius law is not any more valid and the response of the material to stress can be non-linear.

On the contrary, secondary relaxation processes are linear and obey to the Arrhenius law. Some of them can be interpreted in terms of movement of well defined structural elements (for exemple : jump of alcaly ions in silica-alcaly oxyde glasses); others are not clearly explained : for instance, just before the glass transition, a peak or an anomaly in the spectrum internal friction versus temperature more or less resolved from the increase of damping due to the $\alpha$ relaxation, is often observed. 
This feature seems to be associated to delayed elasticity. We propose that such a behavior corresponds to a second relaxation phenomenon ( $\beta$ relaxation) which is, as $\alpha$ relaxation, characteristic of the vitreous state.

In order to have agreement between the structural models generally admitted for describing the vitreous state and the necessity to have two types of atomic arrangements for explaining the two relaxation phenomena $\alpha$ and $\beta$, we propose the following assumption : whatever the structural model considered, there are planar defects or boundaries in glasses. Then, the $\beta$ relaxation could be due to thermally activated movements of atoms or molecules in the boundaries while the $\alpha$ relaxation should be the consequence of cooperative rearrangements of atoms or molecules between the boundaries.

In a first step, the relaxation models well known in the case of crystalline solids are adapted to the case of glassy materials ; hence, experimental results can be interpreted at less qualitatively.

1. Introduction. - Les solides non cristallins sont caractérisés du point de vue structural par l'absence d'ordre à longue distance dans l'arrangement des atomes ou des molécules, et, du point de vue thermodynamique, par le fait qu'ils ne sont pas en équilibre : en d'autres termes, ils possèdent un excès d'enthalpie libre par rapport au même matériau à l'état cristallin.

Il existe différentes classes de solides non cristallins [1]. Nous ne nous intéresserons ici qu'aux solides vitreux que l'on peut définir ainsi : ils sont obtenus à partir de l'état liquide et l'excès d'enthalpie libre résulte d'un refroidissement suffisamment rapide pour que la cristallisation n'ait pas le temps de se produire. En d'autres termes, un verre est un liquide surfondu figé [2]. Au cours du refroidissement, à la température de figeage, ou température de transition, et à l'échelle de temps correspondant aux expériences mises en œuvre pour l'observer, on peut dire qu'il y a quasi-disparition de la mobilité des atomes et molécules permettant de passer d'un état de configuration à un autre. Cette diminution du nombre de degrés de liberté à $T_{0}$ se traduit par une quasi-discontinuité dans la variation de diverses propriétés macroscopiques (densité, chaleur spécifique, coefficient de dilation linéaire, compressibilité...).

On voit donc que l'analyse structurale d'un verre, c'est-à-dire de son état de configuration est indissociable de l'étude de la mobilité des atomes, ions et molécules dans les solides vitreux aux environs de la température de transition. Un exemple d'une telle étude est représenté par les travaux de Johari [3] portant sur les mesures de relaxation diélectrique effectuées sur divers systèmes vitreux ; on observe 2 phénomènes de relaxation : l'un (phénomène $\alpha$ ) correspond à la mise en mouvement de la quasi-totalité des atomes et molécules constituant le verre ; l'autre (phénomène $\beta$ ) semble résulter de la mobilité de certains atomes ou molécules seulement. Seul le $2^{\mathrm{e}}$ phénomène obéit à la loi d'Arrhénius alors que le premier se comporte comme l'indique la relation :

$$
v=v_{0} \exp \left(-\frac{A}{T-T_{0}}\right)
$$

qui s'apparente à l'expression de Vogel-FulcherTammann.

Etant donné la nature diverse des composés étudiés
Johari considère que ces 2 phénomènes de relaxation sont caractéristiques de la structure de l'état vitreux. Il adopte donc l'hypothèse des aggrégats amorphes [4] et la mobilité des molécules diffère alors suivant qu'elles se trouvent dans l'aggrégat, c'est-à-dire dans un domaine de forte densité (relaxation $\alpha$ ) ou qu'elle se trouve entre les aggrégats c'est-à-dire dans un domaine de plus faible densité (relaxation $\beta$ ).

Une autre technique nous paraît pouvoir apporter des éléments d'information intéressants : la mesure du frottement intérieur. Nous nous proposons donc de passer d'abord en revue les divers résultats obtenus quant au frottement intérieur des solides vitreux (partie 2); nous nous limiterons au cas des mesures effectuées à basse fréquence et verrons, successivement, les verres minéraux, les verres métalliques, les solides covalents vitreux et les verres organiques.

Nous évoquerons ensuite (partie 3) les modèles structuraux généralement proposés pour décrire l'état vitreux en montrant comment ils peuvent être complétés par la notion de défauts (en particulier du type interfaces); cette partie sera suivie (partie 4) par la présentation de modèles de relaxation mécanique cohérents avec cette hypothèse.

Enfin, les idées proposées dans la partie précédente seront discutées (partie 5) en faisant référence aux résultats expérimentaux réunis dans la partie 2 .

\section{Frottement intérieur (basse fréquence) des solides} vitreux : résultats expérimentaux. -2.1 VERRES MINÉRAUX. - Nous nous limiterons au cas des verres à base d'oxydes. Les propriétés inélastiques de ces solides ont donné lieu à de nombreuses publications depuis l'un des premiers travaux [5] jusqu'aux revues bibliographiques les plus récentes $[6,7,8,9,10,11]\left({ }^{1}\right)$.

Les verres sont formés de 2 types d'oxydes: un oxyde formateur (typiquement $\mathrm{SiO}_{2}$ ) et un oxyde modificateur (typiquement $\mathrm{Na}_{2} \mathrm{O}$ ). La structure d'un verre formé du seul oxyde formateur correspond à un réseau tridimensionnel de liaisons dirigées (exemple: la

(1) Des sels minéraux ou des mélanges de sels peuvent, à partir de l'état fondu se présenter à l'état vitreux $[3,12,13]$. Les travaux portant sur de tels systèmes sont, à l'heure actuelle, trop peu répandus pour que nous puissions en tenir compte dans cette revue bibliographique. 
silice amorphe résulte de la reproduction non régulière dans l'espace du polyèdre de liaisons covalentes

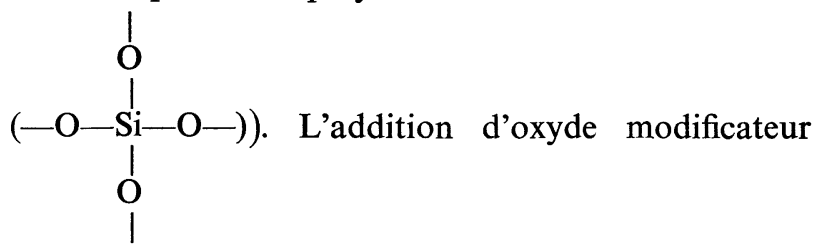
entraîne la rupture de liaison (exemple

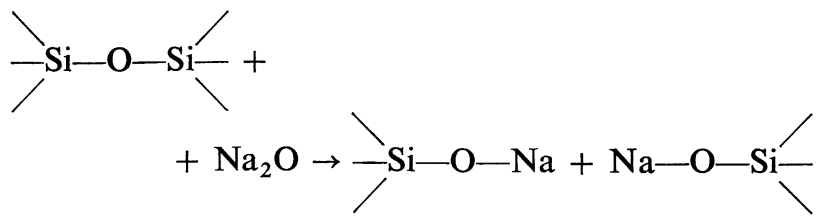
et apparition d'ions oxygène dits non pontants.

Dans ce qui suit, nous évoquerons d'abord le cas des verres $\left(\mathrm{SiO}_{2}\right)_{x}(\mathrm{MeO})_{y}$ (Me étant un métal alcalin). Nous verrons ensuite l'effet de l'addition d'un $3^{\mathrm{e}}$ oxyde.

Dans un $3^{\mathrm{e}}$ paragraphe, nous citerons quelques travaux relatifs à d'autres systèmes (borates, phosphates...). Enfin, nous essayerons de dégager les caractéristiques communes du comportement inélastiques des verres à base d'oxydes.

2.1.1 Verres silicates-alcalins. - On observe 3 types de phénomènes de relaxation :

- L'un dû à des distorsions du réseau de liaisons formant le solide vitreux et généralement étudié aux fréquences élevées [15, 14].

- Un deuxième résultant de sauts thermiquement activés d'ions dans le verre.

- Enfin, un troisième type de relaxation associé à la mobilité de l'ensemble des ions ou groupes d'atomes, apparaissant vers la transition vitreuse.

Dans le cas des verres silicates alcalins, ces 2 derniers processus de relaxation auxquels nous nous limiterons, se traduisent par le spectre schématique de la figure 1 pouvant correspondre au cas $\mathrm{Me}_{2} \mathrm{O}, 3 \mathrm{SiO}_{2}, \mathrm{Me}$ étant $\mathrm{Li}, \mathrm{Na}, \mathrm{K}$ ou $\mathrm{Rb}$ [16].

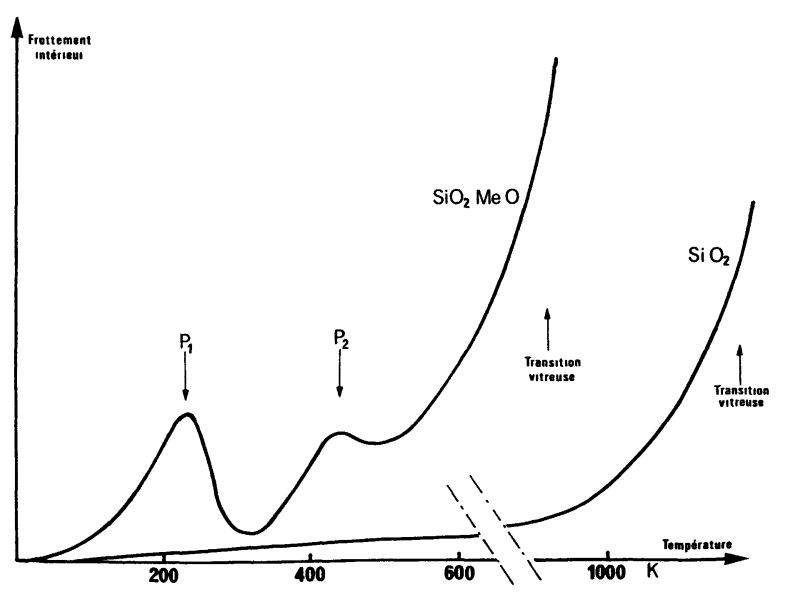

Fig. 1. - Allure schématique des spectres $\delta=f(T)$ des verres binaires. Comparaison avec la silice vitreuse.
Le premier pic de frottement intérieur $P_{1}$ observé vers $240 \mathrm{~K}$ à $1 \mathrm{~Hz}$ n'existe pas dans la silice vitreuse $\left({ }^{2}\right)$ et n'apparaît qu'après addition d'un oxyde alcalin. Sa hauteur augmente avec la teneur en oxyde alcalin comme l'indique la figure 2 .

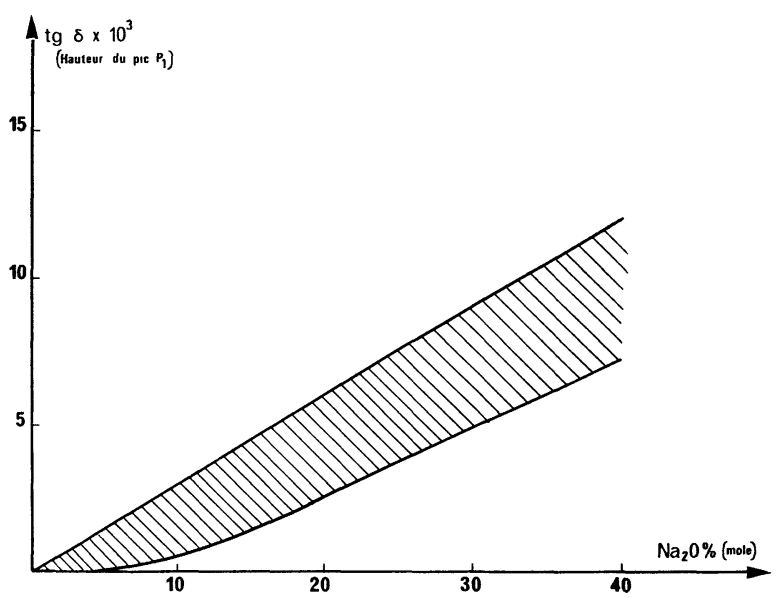

Fig. 2. - Variation de la hauteur du pic $P_{1}$ avec la teneur en $\mathrm{Na}_{2} \mathrm{O}$ (la bande hachurée rassemble les résultats mentionnés par Hopkins et Kurkjian [8]).

En outre, l'énergie d'activation correspondant au pic $P_{1}$ est comparable quelque soit la nature de l'oxyde alcalin à la valeur issue de mesures de conductivité électrique ou de diffusion atomique $[8,10]$. On a donc été conduit à admettre que ce pic $\mathrm{P}_{1}$ était la conséquence du saut thermiquement activé des ions alcalins sous l'effet des contraintes appliquées lors de la mesure du frottement intérieur.

Le $2^{\mathrm{e}}$ pic, $\mathrm{P}_{2}$ apparaissant vers $450-500 \mathrm{~K}$, n'existe, comme $P_{1}$ qu'en présence d'oxyde alcalin et présente une hauteur qui est également proportionnelle à la teneur en oxyde alcalin. Mohyuddin et Douglas [19] ont émis l'hypothèse que ce pic résultait du mouvement des ions oxygène liés à un seul Si. En fait, l'interprétation de ce phénomène de relaxation a donné lieu à de longs débats (voir discussion référence [16]) entre les théories expliquant l'origine du pic $\mathrm{P}_{2}$ par le mouvement des oxygènes non pontant et celles invoquant le mouvement coopératif de complexes formés avec l'ion alcalin $\mathrm{Me}^{+}(\mathrm{Me}-\mathrm{Me}, \mathrm{MeH}, \mathrm{MeOH})$. Il semble toutefois que d'une manière ou d'une autre, le rôle direct ou indirect des oxygènes non pontants soit bien établi.

De même, l'eau, toujours présente dans le verre, est certainement impliquée dans le processus conduisant au pic $\mathrm{P}_{2}$ [21-22].

Notons que ces 2 pics sont bien caractéristiques de l'état vitreux car la dévitrification entraîne leur disparition comme le montre la figure 3 .

(2) Dans le même domaine de température, la silice vitreuse ne donne pas lieu à ces pics de frottement intérieur $[8,17]$; seule apparaît la montée de frottement intérieur vers la transition vitreuse avec une anomalie mal résolue vers 950-900 K [18]. 


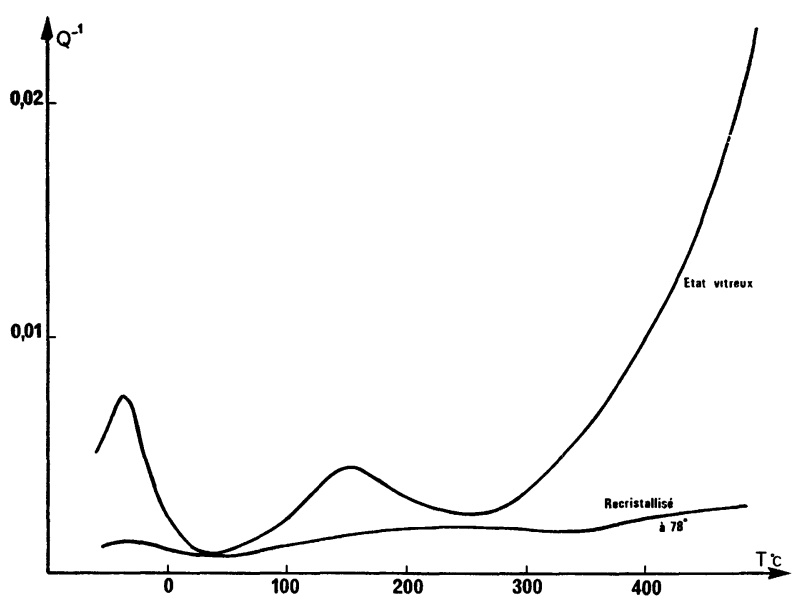

Fig. 3. - Comparaison des spectres $\delta=f(T)$ de mélanges $\mathrm{Li}_{2} \mathrm{O}-2,75 \quad \mathrm{SiO}_{2}$ à l'état vitreux ou recristallisés à $78 \%$ (d'après [23]).

Enfin, on a pu constater que les ions alcalins peuvent aussi donner lieu à une diffusion à longue distance dans un gradient de contrainte [24] ; cet effet devrait pouvoir donner lieu à des phénomènes de relaxation analogue à l'effet Gorski [25].

Nous allons maintenant présenter plus précisément le phénomène responsable de la montée rapide de frottement intérieur vers le domaine de transition. Dans ce domaine, la viscosité du verre diminue considérablement et le comportement du matériau est alors souvent décrit par le modèle rhéologique simple de Maxwell, bien qu'il faille, évidemment, envisager une distribution des temps de relaxation [9]. Un tel modèle rhéologique indique effectivement que le frottement intérieur doit augmenter très rapidement avec la température mais il faudrait connaître la loi de distribution des temps de relaxation pour disposer d'une relation décrivant le frottement intérieur. Une autre méthode a été utilisée : de nombreuses études [8] de relaxation de contrainte ont conduit à utiliser la relation empirique :

$$
\sigma=\sigma_{0} \exp -\left(\frac{t}{\tau_{\mathrm{r}}}\right)^{b}
$$

avec

$$
\tau_{\mathbf{r}}=\tau_{0} \exp \frac{E}{k T}
$$

et $b$, paramètre variant de 0,2 pour des valeurs faibles de $t / \tau_{\mathrm{r}}$, à 1 pour des valeurs élevées de $t / \tau_{\mathrm{r}}$.

A partir d'une telle relation, Douglas et al. [26] calculent l'expression suivante :

$$
\operatorname{tg} \delta=\frac{\pi b}{2^{3 b+1}}\left(\frac{P}{\tau_{\mathrm{r}}}\right)^{b}
$$

$P$ étant la période d'oscillation de leur installation de mesure du frottement intérieur. Ils confirment bien la valeur de $b$ à partir de mesures du frottement intérieur en fonction de la période. En fait, la situation n'est pas aussi simple : dans ce domaine de température, les verres à base d'oxydes présentent une inélasticité qui résulte, d'une part, d'un comportement visqueux, mais d'autre part, également, d'un comportement lié à des phénomènes d'élasticité retardée $[8,27]$ qui pourrait conduire $\left({ }^{3}\right)$ à des maxima de frottement intérieur au-delà du pic $\mathrm{P}_{2}$.

2.1.2 Addition d'un $3^{\mathrm{e}}$ oxyde. - L'addition d'un autre oxyde alcalin à un verre silicate - oxyde alcalin modifie notablement le spectre dans le domaine comprenant les pics $P_{1}$ et $P_{2}$ comme le montre la figure 4 [28].

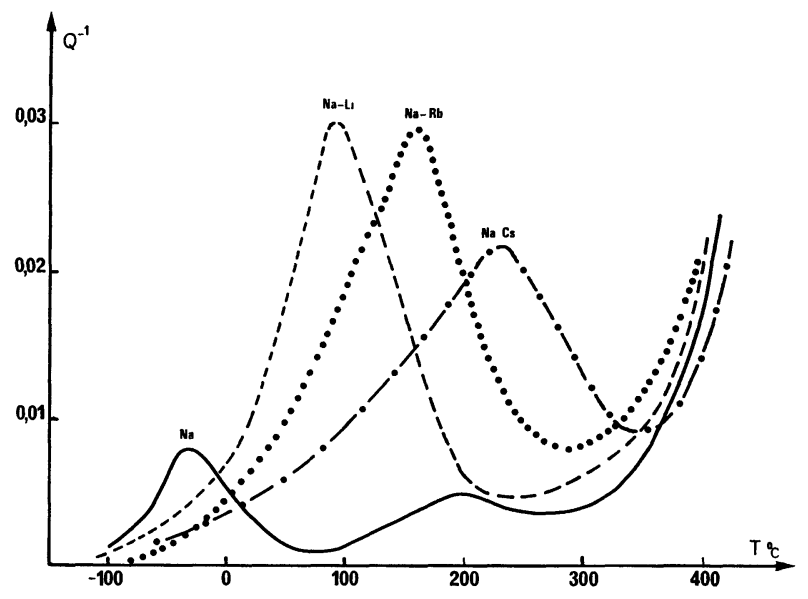

Fig. 4. - Effet du remplacement de la moitié de l'oxyde alcalin par une quantité égale d'un $2^{\mathrm{e}}$ oxyde alcalin (d'après [28]).

Ces modifications sont rappelées dans l'article de synthèse de Day [11] et peuvent se résumer de la façon suivante [29]: quand la teneur en $2^{\mathrm{e}}$ oxyde alcalin croît :

- Le pic $P_{1}$ se déplace vers les hautes températures et sa hauteur décroît.

- Un autre pic $P_{3}$ apparaît entre $P_{1}$ et $P_{2}$, à une température d'autant plus basse et d'une amplitude d'autant plus élevée que le rapport des concentrations en ions alcalins est élevé.

- Le pic $P_{2}$ se déplace vers les températures élevées ; il est difficile d'observer l'évolution de sa hauteur, car il est vite noyé dans le pic $\mathbf{P}_{3}$.

Le pic $P_{3}$ est généralement interprété en envisageant des mouvements coopératifs de complexes formés par les 2 catégories d'ions alcalins. Cette interprétation pourrait être confortée par la corrélation qui apparaît entre la hauteur du pic $P_{3}$ et la valeur du coefficient de diffusion de l'ion alcalin le plus lent [30]. La figure 5 montre en effet que le pic $\mathrm{P}_{3}$ présente une hauteur maximale quand le coefficient de diffusion de l'ion alcalin le plus lent est le plus élevé.

(3) Il est peu pensable que l'élasticité retardée décrite dans la littérature puisse provenir exclusivement des processus physiques à l'origine des pics $P_{1}$ et $P_{2}$. 


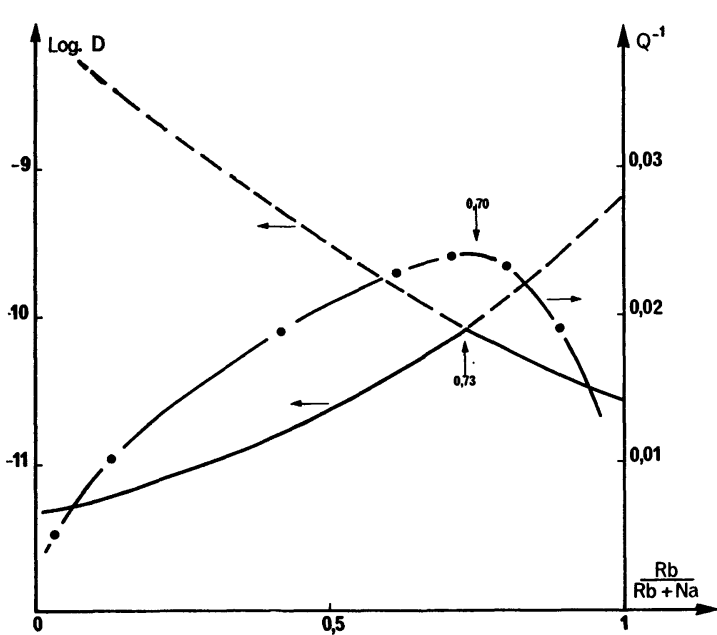

Fig. 5. - Variation du coefficient de diffusion de $\mathrm{Na}^{+}$(courbe décroissante) et de $\mathrm{Rb}^{+}$(courbe montante) avec le rapport $\mathrm{Rb} /(\mathrm{Rb}+\mathrm{Na})$. Comparaison avec l'évolution de la hauteur du pic $P_{3}$ (d'après [30] ).

Une discussion détaillée portant sur les caractéristiques du pic $\mathrm{P}_{3}$ a été développée par Van Ass [31].

Signalons, enfin, que l'addition d'eau semble jouer un rôle analogue à celui de l'addition d'un deuxième oxyde alcalin [21, 22].

2.1.3 Verres plus complexes. - Le comportement inélastique des verres où l'oxyde formateur est soit $\mathrm{P}_{2} \mathrm{O}_{5}[32,33]$ soit $\mathrm{B}_{2} \mathrm{O}_{3}$ [34-35] est très comparable à celui des verres à base de silicate. Toutefois, le pic $\mathrm{P}_{2}$ n'est pas toujours observé dans le cas des verres à base de $\mathrm{P}_{2} \mathrm{O}_{5}[32,33,36]$.

Les verres silicate-oxyde alcalin-oxyde alcalinoterreux ne présentent que le pic $\mathrm{P}_{1}$ à une température qui augmente avec la teneur en oxyde alcalinoterreux. Par contre, un maximum apparaît vers le début de la montée de frottement intérieur liée au domaine de transition. Ce maximum serait lié à la formation d'amas [37].

Enfin, mentionnons le cas des verres silicates-alu-

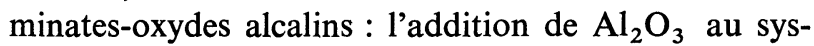
tème $\mathrm{Na}_{2} \mathrm{O}, \mathrm{SiO}_{2}$ fait disparaître le pic $\mathrm{P}_{2}$ [20,38]. (Ce fait a d'ailleurs renforcé l'idée que les oxygènes non pontants sont à l'origine du pic $\mathrm{P}_{2}$.) Mais, par ailleurs, il est possible avec ces verres de faire apparaître le pic $\mathbf{P}_{3}$ soit par addition d'un $2^{\mathrm{e}}$ oxyde alcalin [30] soit même par introduction de protons [40].

2.1.4 Conclusions. - De ces nombreux résultats expérimentaux, nous pouvons dégager les conclusions suivantes : tous les verres à base d'oxyde présentent une montée rapide de frottement intérieur vers le domaine de transition : c'est une manifestation liée au phénomène de relaxation primaire commun à tous les solides vitreux. La loi d'Arrhénius ne peut s'appliquer de façon satisfaisante et, d'autre part, des phénomènes de non linéarité sont signalés. En outre, il existe des effets de relaxation secondaire dont la compréhension en terme de structure et de mouvement d'ions ou de complexes va décroissant dans l'énumération qui suit :

- Le pic $P_{1}$ résultant du saut thermiquement activé d'ions alcalins.

- Le pic $P_{3}$ mettant en jeu 2 types d'ion alcalin.

- Le pic $P_{2}$ à l'origine duquel les oxygènes non pontants et (ou) les protons pourraient jouer un rôle.

- Divers maxima (le plus souvent mal résolus de la montée de frottement intérieur liée à la transition vitreuse) dont l'origine n'est pas clairement établie et qui semblent associés aux effets d'élasticité retardée. Il ne peut donc être exclu qu'existe, dans ce domaine, la relation $\beta$.

2.2 VerRes mÉtAlliQues. - Les verres métalliques offrent un grand intérêt scientifique car leurs propriétés présentent des caractéristiques aussi curieuses qu'inattendues [41]. Par, exemple, bien qu'ils soient formés d'atomes disposés au hasard, leur densité diffère peu de celle du même composé à l'état cristallin; ils sont élastiques, avec un module plus élevé que dans le cas des verres à base de silicate mais ne sont pas fragiles et possèdent en outre une grande aptitude à se déformer plastiquement ; enfin, certains verres métalliques à base de fer ont une dureté aussi élevée que celle d'aciers martensitiques et forment pourtant des solides ferromagnétiques doux.

Chen et Turnbull [42] ont montré que ces matériaux sont, comme les autres solides vitreux, l'objet d'une transition vitreuse dans un domaine de température $\mathrm{Tg}$ au-dessus duquel ils deviennent très facilement déformables ; la viscosité de ces alliages peut, en effet, atteindre des valeurs faibles $\left(2 \times 10^{7}\right.$ poises $)$ avant l'apparition de la dévitrification. On doit donc attendre une augmentation du frottement intérieur $Q^{-1}$ dans le domaine de transition vitreuse. Chen et al. [43] ont étudié les propriétés inélastiques de l'alliage $\mathrm{Pd}_{0,76} \mathrm{Au}_{0,075} \mathrm{Si}_{0,165}$ pour lequel $T_{\mathrm{g}} \# 370^{\circ} \mathrm{C}$. Il apparait bien au-dessus de $200{ }^{\circ} \mathrm{C}$ une telle augmentation de $Q^{-1}$ (figure 6) ; cette augmentation est d'ailleurs

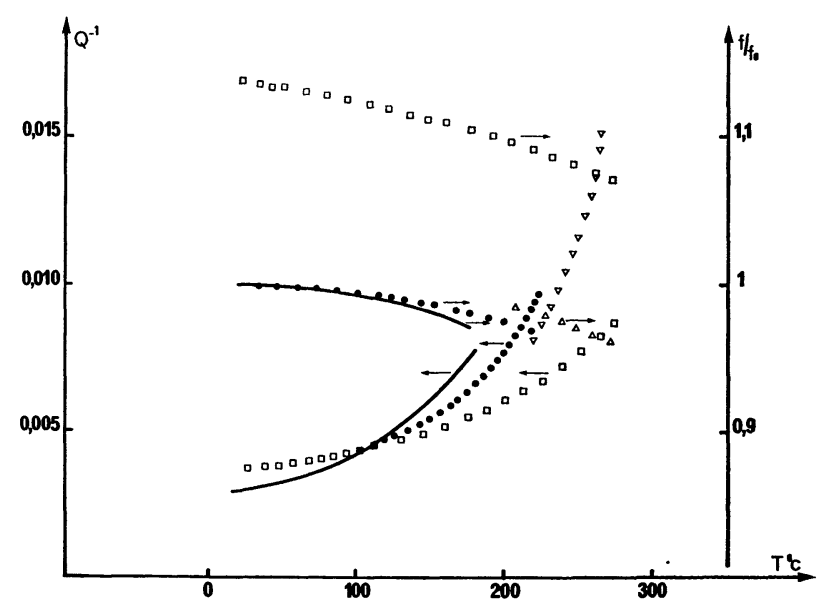

Fig. 6. - Spectres $\delta=f(T)$ d'un verre métallique $\mathrm{Pd}_{0,76} \mathrm{Au}_{0,075} \mathrm{Si}_{0,165}$ (d'après [43]). 
sensible aux traitements thermiques effectués entre 190 et $320^{\circ} \mathrm{C}$. Malheureusement, les mesures étant toutes faites à des températures restant inférieures à $270^{\circ} \mathrm{C}$, il n'est pas possible d'affirmer qu'une telle montée du frottement intérieur se confirme vers $T_{\mathrm{g}}$. Les auteurs interprètent cette manifestation inélastique comme la conséquence d'un phénomène de relaxation se produisant dans des domaines moins compacts du matériau vitreux, ces domaines s'éliminant lors des traitements de recuit. Une telle hypothèse est cohérente, d'une part, avec la large distribution dans la valeur de l'enthalpie d'activation et, d'autre part, avec l'augmentation des constantes élastiques après traitement thermique.

Eisenberg et Reich [44] ont effectué une étude sur le même type d'alliage mais avec des mesures de frottement intérieur faites jusque vers la température de transition vitreuse. Un exemple de leurs résultats est représenté sur la figure $7\left(\right.$ alliage $\left.\mathrm{Pd}_{80} \mathrm{Si}_{20}\right)$ : il est à

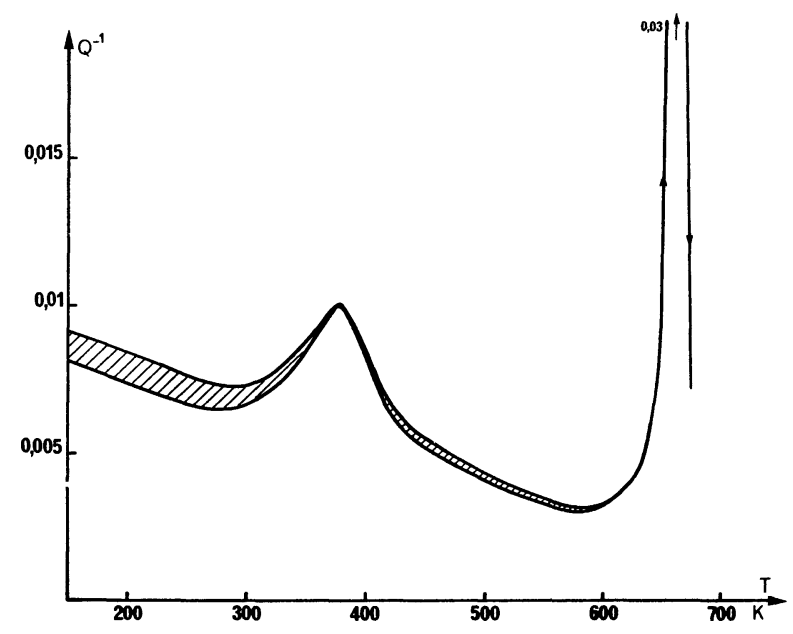

FIG. 7. - Spectre $\delta=f(T)$ d'un verre métallique $\mathrm{Pd}_{80} \mathrm{Si}_{20}$ (d'après [44]).

noter qu'un tel résultat diffère notablement de celui porté sur la figure 6 : fond continu plus élevé et apparition de 2 maxima; l'existence du premier maximum sur la figure 7 entraîne une décroissance du frottement intérieur quand la température augmente entre 120 et $250^{\circ} \mathrm{C}$ alors que l'amortissement varie en sens inverse dans le cas de la figure 6.

Toutefois, Eisenberg et Reich n'observent pas une reproductibilité satisfaisante et attribuent cette diversité des résultats à des phénomènes de séparation de phase lors de l'élaboration des échantillons. Des résultats analogues avec la même difficulté, quant à la reproductibilité, sont obtenus avec des alliages Pd-Au-Si.

Le nombre de travaux portant sur les mesures de frottement intérieur des alliages métalliques amorphes est encore, à l'heure actuelle, réduit. Aussi, nous faut-il signaler l'article de Chen et Goldstein [45] décrivant le comportement viscoélastique de verres métalliques $\mathrm{Pd}-\mathrm{Ag}-\mathrm{Si}, \mathrm{Pd}-\mathrm{Cu}-\mathrm{Si}$ et $\mathrm{Pd}-\mathrm{Au}-\mathrm{Si}$. Des essais de fluage en tension ont montré que la complaisance de tels alliages comprend trois termes :

$$
J(t)=J_{\mathrm{g}}+J_{\mathrm{d}}(t)+t / \eta
$$

$J_{\mathbf{g}}$ : partie élastique de la complaisance du matériau vitreux (de l'ordre de $1,3 \times 10^{-12} \mathrm{~cm}^{2} /$ dyne)

$J_{\mathrm{d}}:$ partie anélastique

$\eta$ : viscosité en tension (environ 3 fois la viscosité en cisaillement en admettant le matériau incompressible).

Au-delà de la déformation purement élastique, il apparaît donc la possibilité de déformer ces alliages vitreux, soit par réarrangement localisé des atomes (comportement anélastique) soit par réorganisation structurale totale (comportement visqueux). On comprend donc que $J_{\mathrm{d}}$ augmente avec la température pour atteindre une valeur maximale vers $T_{\mathrm{g}}$ au-dessus de laquelle le matériau vitreux ne comporte plus qu'un comportement visqueux.

La partie anélastique de la déformation correspond à une distribution des temps de relaxation et vers $T_{\mathrm{g}}$, le nombre de processus à long temps de relaxation augmente avec la température.

D'autre part, on observe un comportement non linéaire à contrainte croissante lorsqu'on approche de $T_{\mathrm{g}}$. En utilisant, une relation du type :

$$
\dot{\varepsilon}=\dot{\varepsilon}_{0} \exp \left(-\frac{\Delta E}{R T}\right) \sinh \frac{\sigma V_{a}}{2 k t}
$$

avec $\Delta E$ énergie d'activation

$V_{a}$ : volume d'activation.

Chen et Goldstein [45] calculent des valeurs de $V_{a}$ variant d'environ un volume atomique bien en dessous de $T_{\mathrm{g}}$ à plus de 100 volumes atomiques au-dessus de $T_{\mathbf{g}}$.

2.3 Solides vitreuX SEMI-CONDUCTEURS. Comme dans le cas des métaux amorphes, peu de travaux ont été publiés dans le domaine du frottement intérieur des solides vitreux semi-conducteurs Imaoka et Sakamura [46] ont effectué des mesures avec un pendule de torsion sur des verres chalcogénures : la figure 8 montre déjà dans le cas du sélénium, que

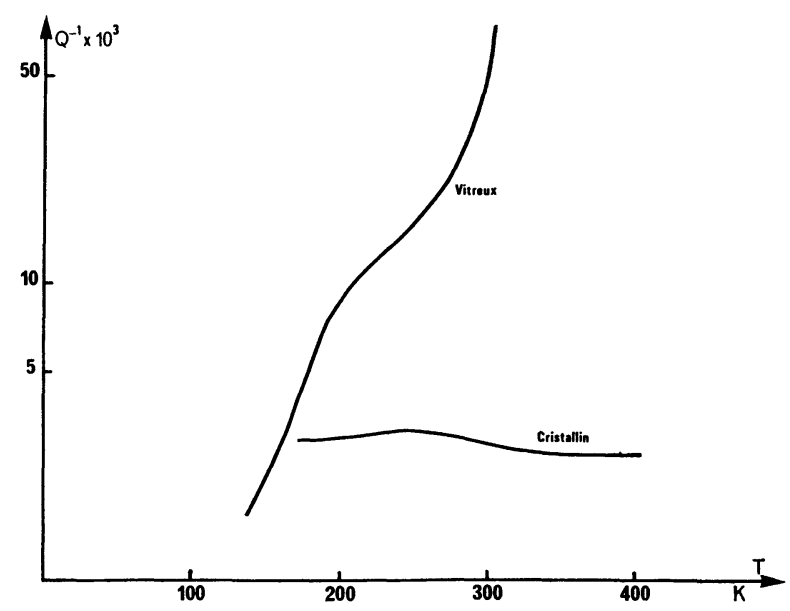

FIG. 8. - Spectre $\delta=f(T)$ du sélénium ; comparaison entre l'état vitreux et l'état cristallin (d'après [46]). 
l'état vitreux se traduit par un frottement intérieur variant bien plus rapidement avec la température que dans le cas de l'état cristallin et cela d'autant plus nettement que l'on approche de la température de transition qui est de l'ordre de 300 à $310 \mathrm{~K}$ [47]. En outre, une anomalie est apparente entre 220 et $270 \mathrm{~K}$.

L'addition d'arsenic ou de germanium provoque un déplacement vers les hautes températures de la partie fortement dépendante de la température; il est à noter que ce déplacement est tout à fait comparable à l'augmentation de $T_{\mathrm{g}}$. La figure 9 montre le cas de

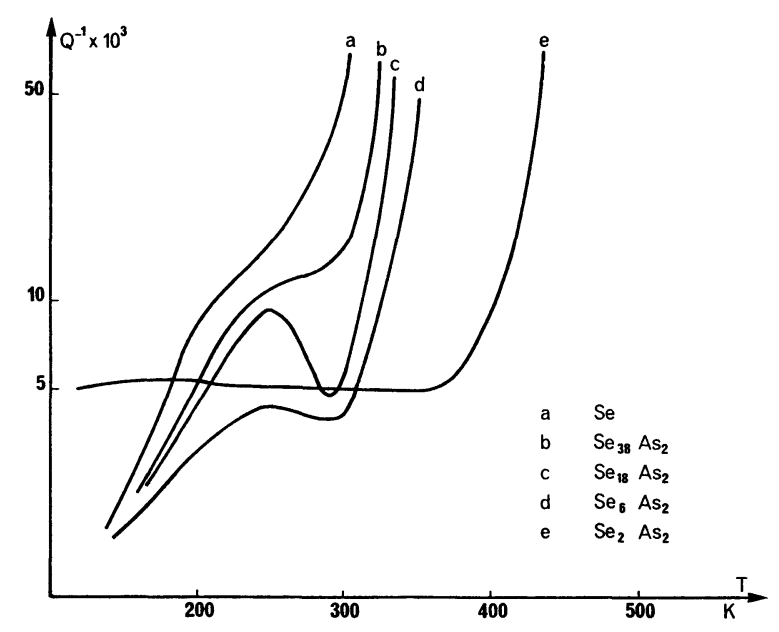

Fig. 9. - Spectres $\delta=f(T)$ de divers mélanges binaires Se-As (d'après [46]).

l'alliage Se-As. Un résultat analogue est observé dans le cas du soufre auquel est ajouté de l'arsenic. Des alliages ternaires ont également été étudiés mais les résultats diffèrent plus d'un alliage à l'autre: par exemple, l'addition de tellure au mélange $\mathrm{As}_{2} \mathrm{Se}_{3}$ ne modifie pratiquement pas le spectre alors que l'addition d'iode au même alliage entraîne une diminution de $T_{\mathrm{g}}$ (et donc un déplacement vers les basses températures de la partie fortement dépendante de la température) : Imaoka et Sakamura [46] expliquent cet écart en indiquant que l'iode à tendance à former une combinaison chimique avec $A_{\mathrm{s}}$ laissant une phase s'enrichissant en Se alors que Te s'incorpore dans la structure amorphe de façon analogue à un ion modificateur dans un verre d'oxydes sans toutefois posséder la mobilité des ions alcalins (pas de phénomène de relaxation du type $\mathbf{P}_{\mathbf{1}}$ ).

Sans donner d'explication très claire, les auteurs considèrent le pic observé vers $280-270 \mathrm{~K}$, qui a toutes les caractéristiques d'un pic de relaxation, comme la conséquence d'une modification (dont la nature est difficile à préciser) des liaisons $B-B$ (B étant Se ou $S$ ) alors que les atomes $\mathrm{A}$ ( $\mathrm{Ge}$ ou $\mathrm{As}$ ) restent fixes. Mais cette interprétation purement qualitative ne permet pas de comprendre que les caractéristiques du phénomène de relaxation puissent varier dans de fortes proportions quand la fraction d'atomes $\mathrm{A}$ augmente : par exemple l'énergie d'activation et le temps de relaxation limite varient respectivement de $26,5 \mathrm{kcal} / \mathrm{mole}$ et $4,5 \times 10^{-24} \mathrm{~s}$ à $11,3 \mathrm{kcal} / \mathrm{mole}$ et $9,3 \times 10^{-11} \mathrm{~s}$ lorsqu'on passe du mélange $\mathrm{GeSe}_{6}$ à $\mathrm{GeSe}_{18}$.

Amrhein et al. [48] ont étudié une autre famille de verres chalcogénures $\mathrm{Si}_{35} \mathrm{As}_{25-x} \mathrm{Ag}_{x} \mathrm{Te}_{40-y} \mathrm{Se}_{y}$.

Pour $x=y=0$, le spectre frottement intérieurtempérature est analogue à celui du sélénium amorphe : une montée rapide du frottement intérieur vers la température de transition précédée d'une anomalie plus ou moins marquée vers $0,7-0,8 T_{\mathrm{g}}$. La figure 10

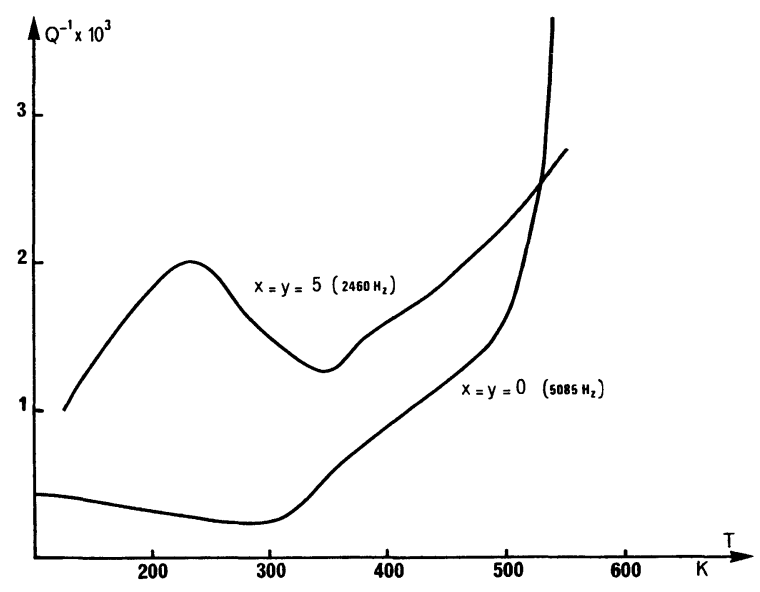

Fig. 10. - Spectres $\delta=f(T)$ de mélanges

$\mathrm{Si}_{35} \mathrm{As}_{25-x} \mathrm{Ag}_{x} \mathrm{Te}_{40-y} \mathrm{Se}_{y}$ (d'après [48]).

montre que pour $x \neq 0$ (addition d'argent) il apparaît un pic de relaxation vers $150-200 \mathrm{~K}$ dont la hauteur est proportionnelle à $x$ et dépend également mais de façon moindre de la teneur en Se $(y)$. L'énergie d'activation (11 à $12 \mathrm{kcal} / \mathrm{mole})$ est comparable à celle issue de mesures de conductivité en courant continu (13 à $15 \mathrm{kcal} / \mathrm{mole}$ ) faites sur des alliages identiques.

Cela conduit Amrheim et al. à considérer ce pic comme la conséquence des mouvements des ions. $\mathrm{A}_{\mathbf{g}}^{+}$dans la structure vitreuse formée par Si-As-Te. En référence aux verres d'oxyde, les ions $\mathrm{Ag}^{+}$joueraient un rôle modificateur alors que $\mathrm{Si}$, As et $\mathrm{Te}$ seraient plutôt formateur.

L'addition de sélénium provoquerait la formation d'un verre biphasé ; la modification des caractéristiques de la relaxation des ions $\mathrm{Ag}^{+}$serait alors simplement la conséquence du comportement différent de ces ions dans chacune de ces phases.

La montée rapide du frottement intérieur, généralement observée vers $T_{\mathrm{g}}$ doit être associée à des mouvements importants des divers atomes constituant le verre semi-conducteur; Il nous semble donc utile de rappeler quelques résultats relatifs au comportement visqueux de tels matériaux, propriété macroscopique mettant également en œuvre des mouvements importants d'atomes. Cukierman et Uhlman [49] ont, par exemple, étudié l'écoulement visqueux du sélénium amorphe; en associant les résultats issus d'essais 
mécaniques à basse température aux mesures de viscosité faites à haute température, ils parviennent à la courbe reliant $\log \eta$ à $1 / T$ (Fig. 11).

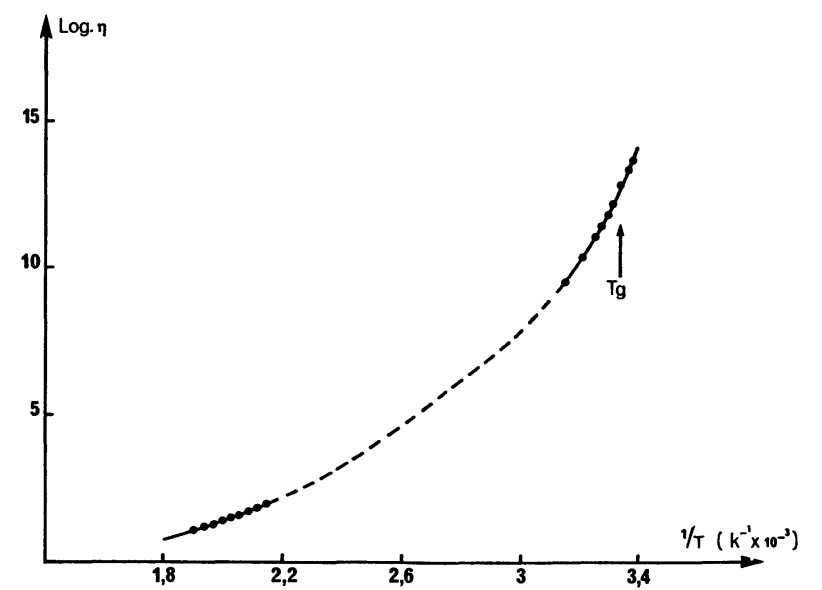

FIG. 11. - Viscosité du sélénium en fonction de la température (d'après [49]).

On voit donc clairement qu'il n'est pas possible d'envisager une loi d'Arrhénius de part et d'autre de $T_{\mathrm{g}}$. En outre, Cukierman et Uhlmann constatent qu'à proximité de $T_{\mathrm{g}}$, leur matériau présente un comportement non newtonien : en effet, la viscosité diminue lorsque la contrainte appliquée augmente. L'application de la relation :

$$
\eta=\frac{\sigma \exp \left(\frac{\Delta E}{k T}\right)}{\dot{\varepsilon}_{0} \sinh \frac{\sigma V_{a}}{2 k T}}
$$

conduit à des valeurs du volume d'activation comprises entre quelques dizaines et la centaine de volumes atomiques.

2.4 VeRRES ORGANIQUES. - Les propriétés viscoélastiques des hauts polymères organiques ont été abondamment étudiées. De nombreuses revues biblio-

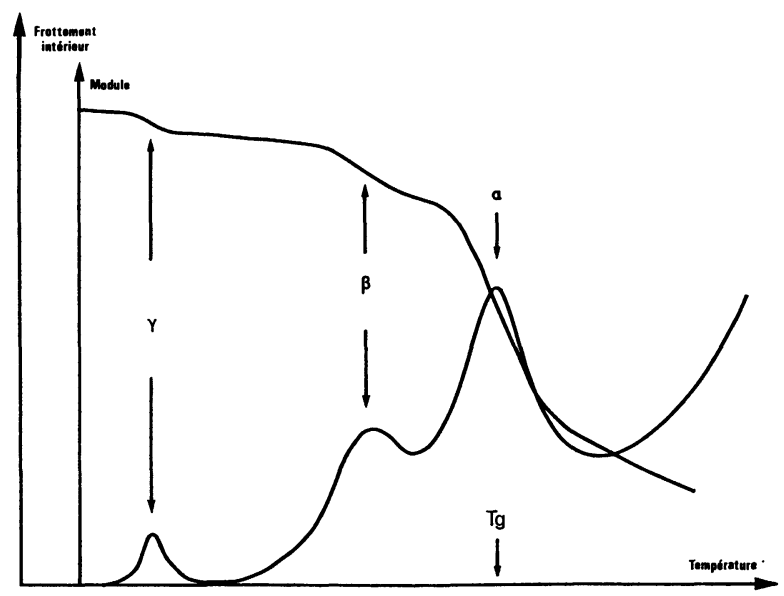

Fig. 12. - Comportement inélastique schématique des verres organiques. graphiques on été proposées [50,51,52]. Nous nous contenterons de rappeler les résultats représentés schématiquement sur la figure 12 , nous limitant ainsi au cas des hauts polymères à l'état vitreux sollicités jusque vers la température de transition.

On distingue ainsi classiquement :

- le processus de relaxation $\alpha$ associé à la transition vitreuse ; le phénomène est caractérisé par une chute importante des constantes élastiques (de $10^{10}$ dynes $/ \mathrm{cm}^{2}$ à $10^{7}$ dynes $/ \mathrm{cm}^{2}$ environ);

- les processus de relaxation secondaires apparaissant à des températures inférieures.

A titre d'exemple, la figure 13 montre le cas du polyméthacrylate avec des radicaux de nature diffé-

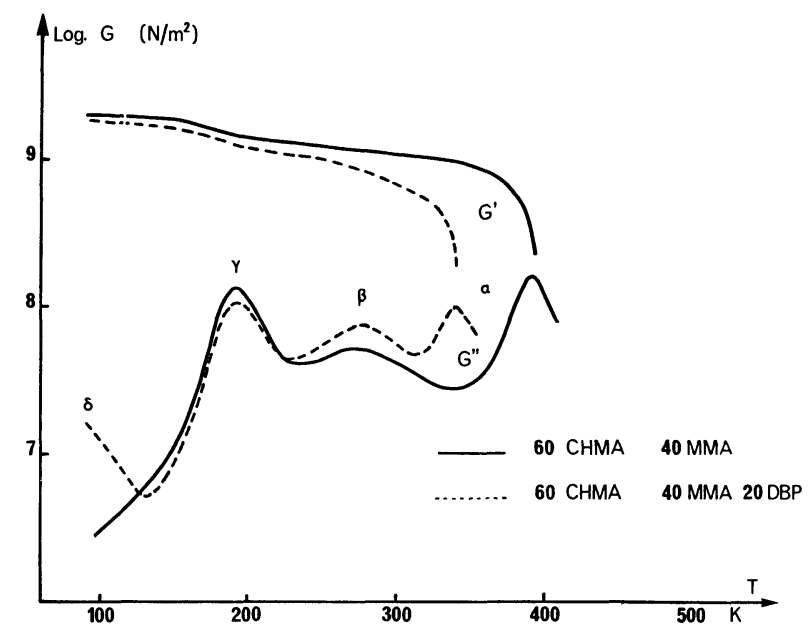

Fig. 13. - Comportement inélastique de 2 mélanges de verres organiques (d'après [53]) contenant du cyclohexyl-méthacrylate, du méthyl-méthacrylate et éventuellement un plastifiant : le dibutyl-phosphate.

rente [53]. On constate la sensibilité du mécanisme $\alpha$ à la nature du radical (variation du coefficient de friction monomérique ou, en d'autres termes du volume libre) et l'existence des processus $\gamma$ et, dans certains cas $\delta$; le pic $\beta$ par contre est peu modifié.

L'interprétation des processus physiques à l'origine de ces différents phénomènes de relaxation est généralement faite en termes de mobilité de certains éléments structuraux. Aussi, outre les mouvements coopératifs mettant en jeu une partie de la chaîne macromoléculaire et se produisant à la température de transition, on évoque $[51,54]$ :

a) Des mouvements de rotation localisés sur la chaîne (positions cis et trans).

b) La rotation de l'ensemble d'un radical autour de sa liaison le rattachant à la chaîne.

c) La rotation d'un radical secondaire autour de sa liaison de rattachant au radical principal.

d) Mouvement de molécules de petite taille (plastifiant dans les volumes libres formés dans la matrice qui constitue le haut polymère vitreux). 
La figure 14 résume de façon schématique ces diverses possibilités.

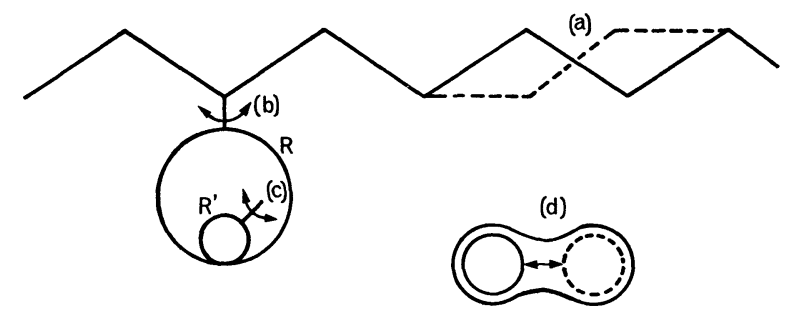

Fig. 14. - Possibilités de mouvement dans une macromolécule : a) changement de configuration d'une portion limitée de la chaîne par rotation de tétraèdres du carbone; $b$ ) rotation du radical $R ; c$ ) rotation du radical $R^{\prime} ; d$ ) mouvement des petites molécules de plastifiant.

La difficulté réside généralement dans l'attribution d'un de ces mécanismes aux divers pics observés.

Contrairement aux phénomènes $\alpha$, les phénomènes de relaxation secondaire obéissent à la loi d'Arrhénius avec une valeur de la fréquence limite, de l'ordre de $10^{13}$, qui semble peu dépendre de la nature du haut polymère $\left({ }^{4}\right)$.

Les résultats signalés ci-dessus correspondent généralement à des comportements linéaires ; toutefois, dans certains cas, on observe des effets non linéaires [51] mais il est difficile d'attribuer ces effets aux processus de relaxation eux-mêmes plutôt qu'à la modification de l'état du matériau sous l'effet de la contrainte (valeur des volumes libres, configuration des chaînes...).

Moins nombreuses sont les études faites sur les verres organiques élaborés avec des composés à bas poids moléculaire. Harrison [56], dans sa revue sur les propriétés viscoélastiques des liquides organiques surfondus signale l'existence de 2 mécanismes de relaxation entre $T_{\mathrm{g}}$ et $T_{\mathrm{F}}$; de même, Barlow et al. [57] interprètent leurs résultats concernant le module de cisaillement complexe de polymères fondus (bas poids moléculaire) en considérant 2 phénomènes de relaxation différents : l'un propre à la structure du liquide surfondu, l'autre lié aux mouvements d'une partie plus ou moins grande de chaque molécule. Des mesures de frottement intérieur à basse fréquence (pendule de torsion) ont confirmé l'existence de phénomènes de relaxation secondaire. Illers [58] a effectué de telles mesures sur des alcools alliphatiques $\left(C_{1}\right.$ à $\left.C_{20}\right)$ et sur des hydro-carbures saturés $\left(\mathrm{C}_{5}\right.$ à $\left.\mathrm{C}_{42}\right)$ solidifiés à l'état vitreux dans une matrice de polystyrène. Faucher et Koleske [59] ont repris ces expériences en mesurant le frottement intérieur d'alcools alliphatiques vitrifiés

(4) Cela permet à Heijboer [54] de proposer la relation $E \mathrm{a}(\mathrm{kcal} / \mathrm{mole})=0,060 \mathrm{Tm}\left(E \mathrm{a}\right.$ : énergie d'activation, $T_{\mathrm{m}}:$ température où est observé le maximum de frottement intérieur à $1 \mathrm{~Hz}$ ) qui est obtenu par l'extrapolation de la méthode de la droite de Wert [55] initialement appliquée au cas des phénomènes de relation dus aux défauts ponctuels dans les cristaux. sur un support de cellulose. Ils retrouvent, pour l'essentiel les résultats de Illers mais observant plus nettement les phénomènes de relaxation secondaire ; un exemple est donné sur la figure $15 a$ et un résultat récent [60] concernant le glycérol amorphe, sur la figure $15 b$.

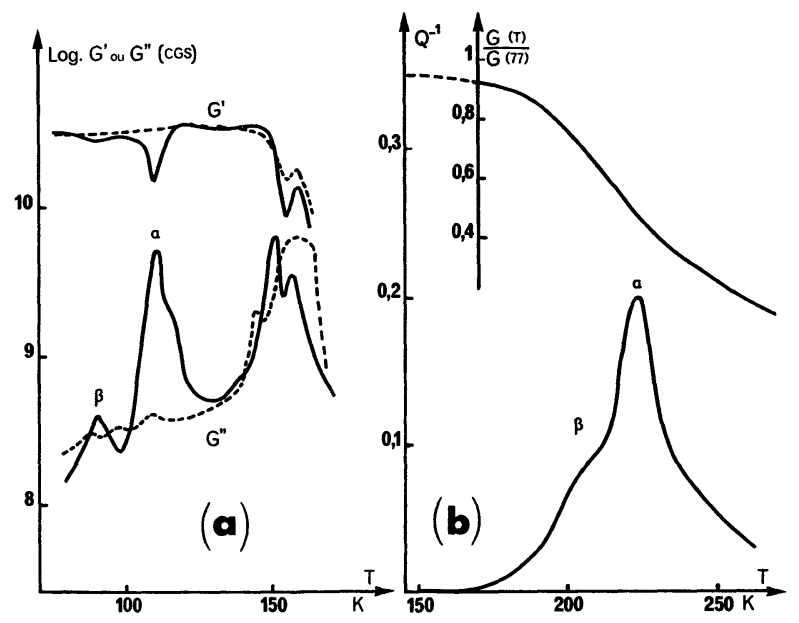

FIG. 15. - Comportement inélastique de verres moléculaires $a$ ) cas du méthanol (d'après [59]); b) cas du glycérol (d'après [60]).

La transition vitreuse se traduit par un pic de frottement intérieur et un minimum de module de cisaillement. En fait, ce type de comportement correspond non seulement à la transition vitreuse mais aussi, d'une part, à l'existence du support de cellulose, d'autre part, à la dévitrification qui se produit, avec ce type de matériau dès que l'on dépasse la température de transition.

Suivant les cas, on observe un ou plusieurs pics correspondant aux phénomènes de relaxation secondaire dont l'origine pourrait être, selon Illers [58] la rotation de certaines parties des molécules alors que celles-ci forment la matrice rigide vitreuse. $\mathrm{Ce}$ type d'interprétation est toutefois mis en doute par Faucher et Koleske [59] pour 2 raisons :

- certains pics secondaires sont observés au-dessus de $T_{\mathrm{g}}$ (cas de l'éthanol);

- les rotations au niveau de molécules petites comme $\mathrm{CH}_{3} \mathrm{OH}$ ou $\mathrm{CH}_{3} \mathrm{CH}_{2} \mathrm{OH}$ apparaissent très probablement à des températures bien inférieures $\left(<-200^{\circ} \mathrm{C}\right)$.

2.5 Conclusions. - De l'ensemble des résultats que nous venons d'évoquer, il ressort les points suivants :

- Vers la température de transition vitreuse, on observe un frottement intérieur augmentant rapidement avec la température (verres moléculaires) ou passant par un maximum (hauts polymères) : c'est une manifestation de la relaxation $\alpha$ mettant en œuvre la quasi-totalité de la structure vitreuse. La loi d'Arrhénius n'est pas respectée et le comportement du matériau n'est pas linéaire vis-à-vis de la contrainte. 
- Au-dessous de $T_{\mathrm{g}}$, il existe divers processus de relaxation secondaire. La plupart d'entre eux sont interprétés de façon satisfaisante en termes de mouvement thermiquement activé de certains éléments structuraux (radicaux, ions...). Il n'en reste pas moins qu'un phénomène de relaxation secondaire (que nous désignerons par relaxation $\beta$ ) semble commun à tous les solides vitreux : il serait donc caractéristique de l'état vitreux.

Dans ce qui suit, nous proposons une interprétation des 2 phénomènes de relaxation $\alpha$ et $\beta$ à partir de considérations sur les modèles structuraux de l'état vitreux.

3. Modèles structuraux. - Bien qu'il faille distinguer les solides non cristallins obtenus par différents procédés [1], on peut, dans le cas des solides vitreux à liaisons dirigées, classer les modèles structuraux en trois catégories [4] :

- modèle microcristallin

- modèle des amas amorphes

- modèle du réseau désordonné.

D'après le premier de ces modèles, le solide vitreux serait constitué de micro-cristaux dont les orientations et les tailles sont distribuées comme elles peuvent l'être dans un solide polycristallin classique ne possédant pas de texture. Ce modèle ne peut avoir de signifification que si la taille des cristallites est au moins égale à 2 ou 3 cellules élémentaires ; elle ne peut cependant guère être supérieure, sinon il ne s'agirait plus d'un solide vitreux mais d'un polycristal à grains très fins. Dans ces conditions, environ la moitié des atomes se trouve dans les joints de grains.

En fait, ce type de modèle est en désaccord avec, par exemple, les résultats de Moss et Graczyk [61] dans le cas du silicium et germanium amorphe. Ces auteurs montrent en effet que le premier pic dans la fonction de distribution radiale correspond précisément à la distance entre premiers voisins dans un cristal; si les joints de grains correspondent à la moitié du volume, le nombre de coordination devrait être dans le cas des premiers voisins, sensiblement inférieur à celui existant dans le cristal parfait.

Dans le modèle d'amas amorphes, on suppose qu'un petit nombre d'atomes (de 10 à 100 environ) peuvent s'arranger en une configuration régulière, de densité élevée (donc de faible énergie potentielle) mais cette configuration ne possède pas les symétries requises pour permettre la triple-périodicité à longue distance et donc la formation du cristal (par exemple, Grigorovici et Manaila [62] invoquent une symétrie d'ordre 5 dans l'amas). La probabilité d'obtenir des amas de taille supérieure est négligeable et le solide est donc constitué d'amas amorphes de petite taille raccordés entre eux par des zones moins denses ou tissus de raccordement [63]. Ce modèle correspond mieux que le précédent, aux fonctions de distribution radiale expérimentales car le mode d'empilement réduit considé- rablement la proportion d'atomes en position de $3^{\mathrm{e}}$ voisin ; il subsiste néanmoins un écart significatif au niveau de la finesse du pic correspondant aux seconds voisins.

Enfin, dans le modèle du réseau désordonné idéal, concept avancé depuis fort longtemps par Zachariassen [64], chaque atome est relié à un nombre de premiers voisins permettant la satisfaction des règles de Valence (il n'existe pas de liaison non satisfaite). Les angles de liaison s'écartent, suivant une certaine distribution, de leur valeur moyenne caractéristique de cristal parfait ; il en est de même, mais avec une distribution plus étroite de la distance entre atomes plus proches voisins. On peut, dans ces conditions accroître fortement les dimensions d'une telle structure sans avoir à couper de liaison ou à augmenter la distorion locale moyenne. De tels modèles ont été utilisés, dans des conditions satisfaisantes, pour simuler la structure de la silice vitreuse [4] du silicium et germanium amorphe [65] ou de verres chalcogénures [66]. L'accord entre ce type de modèle et les fonctions de distribution radiale est excellent; en particulier, la distance aux seconds voisins est en moyenne identique à celle rencontrée dans le cristal mais présente une grande dispersion.

Dans le cas des verres métalliques, on admet que l'on a des arrangements compacts mais désordonnés d'atomes métalliques ; ces arrangements correspondent à la formation de sites, le plus généralement tétraédriques, dans lesquels peuvent se loger des atomes pouvant former des liaisons à caractère covalent [41, 86]. Il se forme ainsi des amas atomiques polytétraédriques.

Examinons maintenant comment ces modèles peuvent permettre l'interprétation qualitative des résultats exposés dans la première partie.

Nous devons tout d'abord souligner que ces résultats, ainsi que ceux de Johari [3] et de Johari et Goldstein [67] obtenus par étude de la relaxation diélectrique de verres moléculaires militent en faveur de la présence de 2 types d'environnement structuraux. En effet, ce type de verres formés de petites molécules ne présente pas les degrés de liberté internes que l'on associe, dans le cas de polymères, aux radicaux ou aux chaînes satellites branchées et que l'on invoque traditionnellement pour interpréter certains processus de relaxation.

Il apparaît cependant, plus ou moins nettement mais de façon quasi générale, qu'au-dessous du domaine de relaxation $\alpha$ correspondant à la transition vitreuse, il existe un domaine de relation $\beta$ situé vers $0,7-0,8 T_{\mathbf{g}}$. Bien que ce processus $\beta$ ne soit pas toujours clairement observé, la diversité de nature des matériaux où il apparaît nous permet raisonnablement de penser, avec Johari [3] Goldstein [63, 68], Williams et Watts [69], qu'il s'agit d'un processus caractéristique de l'état vitreux. Dans ces conditions, il devient nécessaire d'envisager pour les atomes ou molécules 2 types d'environnement correspondant chacun à l'un des processus de relaxation. 
Les 2 premiers modèles (microcristallites et amas amorphes) fournissent l'un et l'autre les 2 types d'environnement requis :

a) un environnement à densité plus faible où la plus grande mobilité des atomes ou des unités moléculaires pourrait permettre l'apparition d'un processus $\beta$ au-dessous de la température de transition,

b) un environnement caractéristique de régions de haute densité avec une mobilité ne pouvant prendre une valeur appréciable conduisant à un effet de relaxation qu'au voisinage de la température de transition.

Cependant, nous venons de voir que ces 2 modèles ne permettent pas d'interpréter aussi correctement que le modèle du réseau désordonné les résultats expérimentaux portant sur les fonctions de distribution radiale. Dans ce dernier modèle, par contre, n'apparaît qu'un seul type d'environnement ce qui semble en désaccord avec les résultats issus des essais de relaxation; cela nous amène à ne retenir que les modèles les plus couramment admis, mais en postulant, en outre, l'existence de défauts du type interface.

On peut en effet s'interroger sur le fait qu'un solide vitreux puisse être constitué ainsi que le suggèrent divers auteurs [4] comme une gigantesque molécule construite presque régulièrement en évitant les liaisons libres et les distorsions trop importantes.

Les expériences de Moss et Graczyk [61] effectuées sur le silicium en film amorphe indiquent l'existence d'une forte concentration en de tels défauts. Brodsky et al. [70] suggèrent en outre que des liaisons libres sont associées à ces défauts qui pourraient être des joints entre amas, joints dans lesquels se trouveraient des atomes présentant des liaisons libres. Pour leur part, Moss et Graczik assimilent ces défauts à des cavités qui s'élimineraient d'ailleurs par recuit, la densité du matériau approchant alors celle du cristal. Même dans ce cas, il est difficile d'admettre que ces cavités disparaissent en laissant un réseau désordonné idéal.

De même, dans le cas des verres métalliques, la notion de défauts linéaires est évoquée $[41,85,87]$. De plus, dans le cadre du modèle de l'arrangement polytétraédrique des atomes [86], peut se poser le problème des «joints » entre les arrangements ; ces 2 remarques rendent plus plausibles l'hypothèse de l'existence d'interfaces au sein du verre métallique.

Le faible volume relatif des interfaces permet à la densité, d'une part, et à la fonction de distribution radiale, d'autre part, de rester en bon accord avec le modèle du réseau désordonné. Par ailleurs, cette approche permet d'envisager deux types d'environnement atomique conduisant aux 2 processus de relaxation $\alpha$ et $\beta$ :

- Dans les interfaces il apparaît que les déplacements d'atomes ou de molécules se font aisément car la probabilité de trouver un volume libre nécessaire au saut d'un atome dans son voisinage immédiat, est grande ; d'ailleurs, la notion de lacune au sens cristallin du terme garde un sens même si une relaxation structurale conduit à considérer cette lacune comme distribuée dans un volume de matière supérieure au volume atomique (voir par exemple la figure 18). Un tel saut d'atome est bien entendu activé thermiquement et entraîne le mouvement de l'interface : ce point sera vu plus en détail dans la partie suivante mais d'ores et déjà on peut prévoir qu'une loi d'Arrhénius doit pouvoir s'appliquer à ces mouvements.

- Entre les interfaces, on est généralement conduit à préférer, pour les mouvements diffusionnels d'atomes ou de molécules, l'hypothèse du réarrangement coopératif. Dans cette hypothèse, la diffusion n'est possible qu'à partir d'une température critique $T_{0}$, proche de $T_{\mathrm{g}}$, et le processus de relaxation qui en résulte présente un temps de relaxation variant comme $\exp \left(-\mathrm{Cte} / T-T_{0}\right)$.

Sur la base de ces remarques, nous allons envisager plus précisément quelques mécanismes permettant la description qualitative (ou semi quantitative) des processus de relaxation $\alpha$ et $\beta$.

4. Interfaces dans les solides vitreux et phénomènes de relaxation. - 4.1 ANALOGIES ENTRE LES MATÉRIAUX VITREUX ET LES SOLIDES CRISTALLINS. La première approche qui vient à l'esprit consiste à s'inspirer des résultats connus dans le cas des solides cristallins en les adaptant aux cas des matériaux non cristallins.

Les relaxations $\alpha$ et $\beta$ sont, du point de vue de l'état vitreux, observées à haute température et il apparaît normal de vouloir les comparer aux phénomènes observés à haute température dans les cristaux.

Par ailleurs, il semble que les processus $\alpha$ et $\beta$ soient de natures proches puisque l'énergie d'activation apparente de la relaxation $\alpha$ tend, à haute température, vers la valeur calculée dans le cas de la relaxation $\beta$. Ce point, représenté sur la figure 16 , a été souligné par Johari [3].

Williams et Watts [69] comme Koppelmann [72]

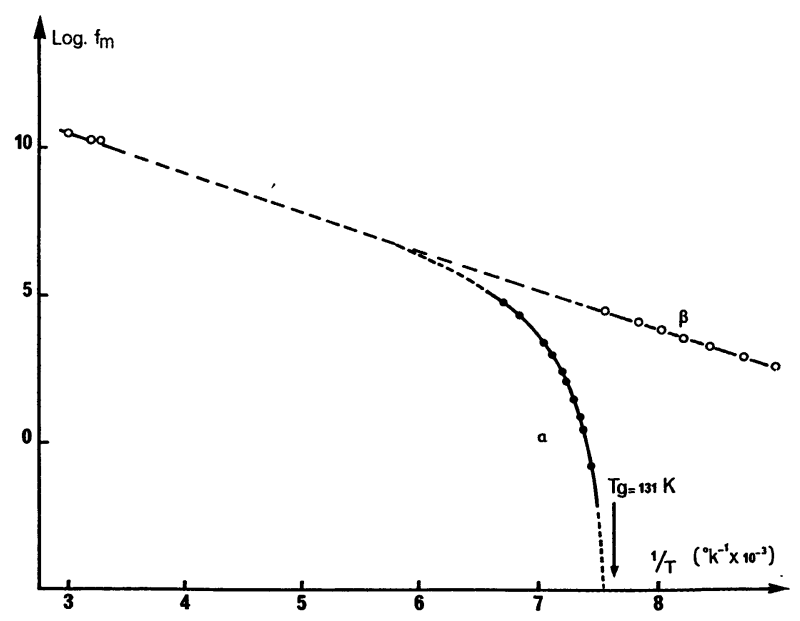

Fig. 16. - Variation de la fréquence avec la température pour les relaxations $\alpha$ et $\beta$ (d'après [3]). 
envisagent même la possibilité d'un couplage entre les processus $\alpha$ et $\beta$ à haute température où il ne subsisterait plus qu'un seul phénomène de relaxation. Enfin, il est possible qu'une relaxation analogue à $\beta$ puisse apparaître bien au-dessus de $\mathrm{T}_{\mathrm{g}}$, dans l'état liquide surfondu.

On peut alors envisager 2 situations possibles pouvant conduire à un double mécanisme de relaxation :

a) dans la structure vitreuse, l'existence de 2 environnements atomiques entraîne 2 types de réorientations possibles des atomes ou groupes d'atomes jouant le rôle de centre de relaxation :

- une réorientation mettant en jeu des déplacements ou variation d'angle de liaison peu importants, réversibles et conduisant à l'effet $\beta$;

- une réorientation avec déplacements ou variation d'angle de liaison notables : on obtient l'effet $\alpha$.

b) Dans la structure vitreuse, l'un des environnements atomiques correspond à une structure peu dense où les atomes ou groupes d'atomes peuvent se réarranger par activation thermique pour atteindre des configurations d'énergie supérieure à celle du même solide avant application de la contrainte. $\mathrm{Si}$ ce réarrangement est couplé avec un champ de force (mécanique, électrique...), il en résulte un effet de relaxation pouvant être décrit en première approximation par le formalisme de Debye, avec en particulier un temps de relaxation obéissant à la loi d'Arrhénius.

L'autre environnement atomique, plus dense, peut donner lieu à des réarrangements coopératifs permettant de passer d'une configuration à une autre configuration de stabilité équivalente ; on a alors une mobilité irréversible des atomes généralement décrite par une loi de Vogel-Fulcher-Tammann (temps de relaxation donné par $\left.\tau_{\mathrm{r}}=\tau_{0} \exp \left(\mathrm{Cte} / T-T_{0}\right)\right)$. Le frottement intérieur qui en résulte ne se présente plus sous forme de pic mais sous forme de croissance monotone avec la température.

Ces deux situations ont leur équivalent dans le domaine des solides cristallins : la première s'apparente, par exemple, au cas des cristaux contenant un défaut dont le nombre de degrés de liberté augmente avec la température : ainsi, ont été signalés le cas de l'oxygène dans le silicium, tel que l'a développé Nowick [73] et celui de défauts interstitiels dans le graphite [74] où 2 pics de frottement intérieur observés après irradiation pourraient résulter du même défaut. La deuxième situation pourrait correspondre au comportement des matériaux polycristallins à haute température : vers 0,4-0,6 $T_{\mathrm{F}}$ apparaît un pic de frottement intérieur non observé dans le cas du même matériau à l'état monocristallin $\left({ }^{5}\right)$.

(5) En réalité, la situation est plus complexe, plusieurs pics sont souvent associés à la présence de joints de grains ; au contraire, il semble possible d'observer un pic de frottement intérieur présentant des caractéristiques comparables dans le cas de monocristaux ayant subis certains traitements thermomécaniques particuliers.
Ce pic est suivi d'une montée rapide du frottement intérieur avec la température. Cette montée peut être due soit à des mouvements de dislocations soit à des processus de diffusion sous contrainte (fluage de Herring-Nabarro).

Les caractéristiques expérimentales des processus $\alpha$ et $\beta$ nous incitent à ne considérer que la $2^{\mathrm{e}}$ situation évoquée ci-dessus :

- phénomène $\beta$ résultant des mouvements atomiques ou moléculaires thermiquement activés au niveau de l'interface ;

- phénomène $\alpha$ dû au mouvement coopératif des atomes ou molécules entre les interfaces.

Dans ces conditions, nous pouvons proposer, pour le phénomène $\beta$ un modèle s'appuyant sur les modèles interprétant le pic de frottement intérieur associé au mouvement des joints de grains dans les solides polycristallins, et, pour le phénomène $\alpha$, un modèle tenant compte de la diffusion sous contrainte. Dans les 2 cas, il sera tenu compte des caractéristiques propres à la structure vitreuse.

4.2 Modèle DE RELAXAtion $\beta$. - D'une manière générale, on peut comprendre l'existence d'un pic de frottement intérieur dû au mouvement d'interfaces en admettant :

- Soit un cisaillement visqueux de cet interface, les joints triples constituant les ancrages justifiant l'existence d'une force de rappel (Fig. 17). La contrainte de cisaillement appliquée sur le joint est relâchée par le

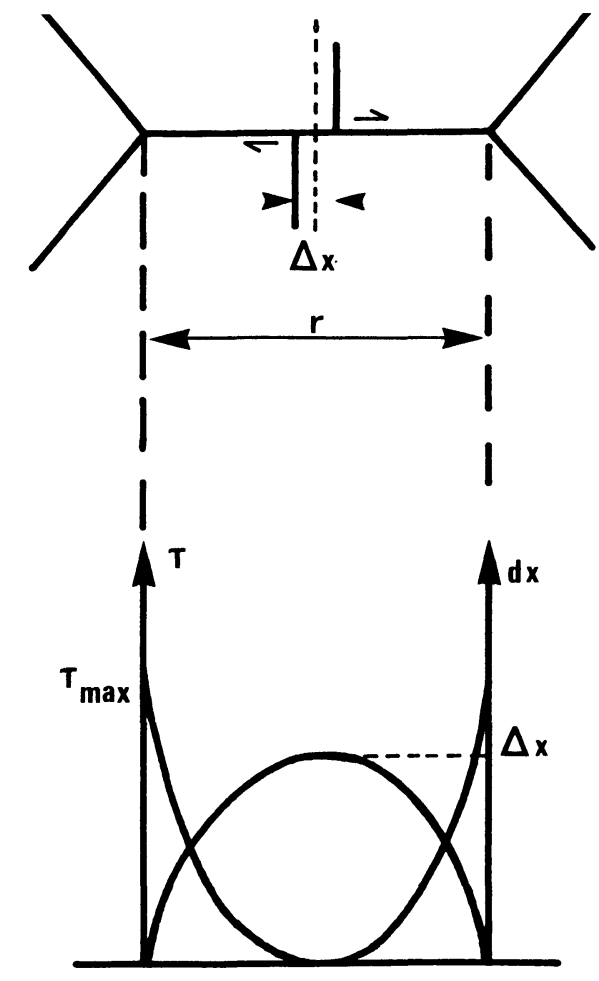

Fig. 17. - Cisaillement au niveau de l'interface avec jonctions entre interfaces fixes ; variation du cisaillement et de la contrainte le long de l'interface. 
déplacement relatif $\Delta x$; cela suppose une contrainte importante dans les zones proches des points triples, d'où la force de rappel.

- Soit un déplacement normal à l'interface favorisant les parties orientées de façon à posséder une complaisance élevée aux dépens des parties à faible complaisance. Ce mécanisme nécessite l'existence d'une anisotropie vis-à-vis de la contrainte.

Nous ne pouvons donc retenir pour le processus $\beta$ que le premier mécanisme, le matériau étant non cristallin et cela impliquant un comportement isotrope vis-à-vis de la contrainte.

Dans ces conditions, il apparaît que sous l'effet d'une contrainte le solide vitreux présente une déformation $\varepsilon$ qui est la somme d'une déformation élastique instantanée et d'une déformation élastique retardée. On se trouve ainsi dans les conditions du solide linéaire idéal où le frottement intérieur est donné par :

$$
\delta=\frac{M_{\mathrm{I}}-M_{\mathrm{R}}}{M_{\mathrm{I}}} \cdot \frac{\omega \tau_{\mathrm{r}}}{1+\omega^{2} \tau_{\mathrm{r}}^{2}}
$$

avec $M_{\mathrm{I}}:$ module instantané,

$M_{\mathrm{R}}$ : module relaxé.

Il faut alors préciser l'intensité de relaxation $\frac{M_{\mathrm{I}}-M_{\mathrm{R}}}{M_{\mathrm{I}}}$ et le temps de relaxation $\tau_{\mathrm{r}}$.

4.2.1 Temps de relaxation. - Curieusement, les premiers modèles de joint se rapprochent dans une certaine mesure, de la conception que nous avons aujourd'hui de l'état non cristallin. Cela provient de l'absence à cette époque de modèle de joints plus raffinés (joints de coïncidence par exemple) et des résultats de microscopie électronique portant sur l'analyse des parois de dislocations.

Ainsi, KE [77] considère le joint comme constitué localement de groupes d'atomes désordonnés contenant un peu moins d'atomes que le même volume de cristal. On rejoint ainsi la notion de lacunes distribuées où $\mathbf{n}-1$ atomes occupent le volume qu'occuperaient n atomes constituant un cristal. Cette notion, qui n'est pas nouvelle puisqu'elle n'est que l'extension du concept de lacune dissociée dans un milieu cristallin constitue en quelque sorte le raccord avec la notion de volume libre. KE envisage alors, comme l'indique la figure 18 , le réarrangement d'un tel groupe d'atomes

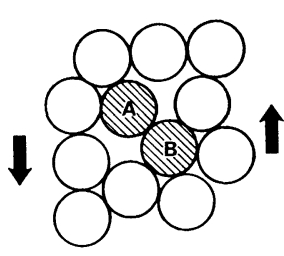

(a)

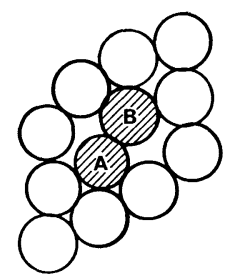

(b)
FIG. 18. - Représentation schématique du mouvement de cisaillement dans le pseudo-joint par échange des atomes $\mathrm{A}$ et $\mathrm{B}$ et réarrangement des atomes premiers voisins. par activation thermique et pouvant entraîner le cisaillement élémentaire $\delta \gamma_{\mathrm{d}}$. Donc la vitesse de cisaillement visqueux sera donné classiquement par

$$
\Delta \dot{\gamma}_{\mathrm{d}}=N r^{2} e \delta \gamma_{\mathrm{d}} v_{0} \exp \left(-\frac{A}{k T}\right) \sinh \frac{B \tau}{k T}
$$

$r$ et $e$ sont respectivement la dimension latérale et l'épaisseur de l'interface.

$A$ et $B$ sont des constantes et $N$, le nombre de groupes d'atomes où peut se produire le cisaillement $\delta \gamma_{\mathrm{d}}$, par unité de volume de joint. Avec $B \tau<k T$, nous sommes dans l'approximation du comportement newtonien et on en déduit le coefficient de viscosité :

$$
\eta_{\mathrm{J}}=\frac{k T}{B N v_{0} \delta \gamma_{\mathrm{d}} r^{2} e} \exp \frac{A}{k T} .
$$

Il semble que l'on puisse adopter ce modèle pratiquement sans modification. Ce formalisme laisse penser que la viscosité associée aux mouvements se produisant au niveau des interfaces s'apparente à la viscosité du même matériau à l'état liquide : cela peut être à l'origine du résultat de Johari [3] rappelé sur la figure 16 ; le mécanisme $\beta$ généralement observé au-dessous de $T_{\mathrm{g}}$ peut exister au-dessus de $T_{\mathrm{g}}$.

Dans l'hypothèse du solide linéaire idéal dans laquelle nous nous sommes placés, la valeur d'équilibre $\overline{\Delta x}$ est proportionnelle à la contrainte et, de plus, quand le solide n'est pas à l'état d'équilibre $\Delta x$ tend vers sa valeur d'équilibre à une vitesse proportionnelle à l'écart :

$$
\frac{\mathrm{d}}{\mathrm{d} t} \Delta x=-\frac{\Delta x-\overline{\Delta x}}{\tau_{\mathrm{r}}} .
$$

Dans ces conditions, il est possible de relier le temps de relaxation à la viscosité de l'interface ; avec $\Delta x=0$, on a :

$$
\frac{1}{2 e} \Delta \dot{x}=\Delta \dot{\gamma}_{\mathrm{d}}=\frac{\tau}{n_{\mathrm{J}}}=\frac{\overline{\Delta x}}{2 e \tau_{\mathrm{r}}} .
$$

D'autre part :

$$
\frac{\overline{\Delta x}}{r / 2} \simeq \frac{\tau}{G_{I}}
$$

Cette relation revient à dire que la contrainte appliquée est relaxée sur une distance de $r / 2$ environ, d'où

$$
\frac{\tau}{n_{\mathrm{J}}}=\frac{\tau r}{4 G_{\mathrm{I}} \tau_{\mathrm{r}} e}
$$

et

$$
\tau_{\mathrm{r}}=\frac{k T}{4 r e^{2} B N G_{\mathrm{I}} v_{0} \delta \gamma_{\mathrm{d}}} \exp \frac{A}{k t}
$$

ou

$$
\tau_{\mathrm{r}}=\frac{n_{\mathrm{J}} r}{4 e G_{\mathrm{I}}}
$$

A partir de ces relations appliquées, par exemple, au 
cas du sélénium et en prenant pour $n_{\mathrm{J}}$ la valeur correspondant à l'état liquide extrapolée jusqu'à la température de relaxation $\beta$, soit $n_{\mathrm{J}}=10^{9}$ à $10^{10}$ CGS [49]. $G_{\mathrm{I}}=4 \times 10^{10}$ dynes $/ \mathrm{cm}^{2}$ et $\tau_{\mathrm{r}} \sim 0,5 \mathrm{~s}$ [78], on peut estimer r compris entre 40 et $400 \AA$.

4.2.2 Intensité de relaxation. - En considérant des grains équiaxes de taille uniforme, Zener [75] à partir de considérations énergétiques, calcule un module de Young relaxé donné par :

$$
E_{\mathrm{R}}=\frac{E_{\mathrm{I}}}{2} \frac{7+5 v_{\mathrm{P}}}{7+v_{\mathrm{P}}-5 v_{\mathrm{P}}^{2}}
$$

$\nu_{\mathrm{P}}$ : coefficient de Poisson;

$\mathrm{KE}$ [76] en déduit la valeur du module de cisaillement relaxé

$$
G_{\mathrm{R}}=\frac{2 G_{\mathrm{I}}}{5} \frac{7+5 v_{\mathrm{P}}}{7+4 v_{\mathrm{P}}}
$$

Ces résultats conduisent à une intensité de relaxation

$$
\frac{G_{\mathrm{I}}-G_{\mathrm{R}}}{G_{\mathrm{I}}} \sim 0,36 .
$$

Il est possible d'aboutir plus simplement à une estimation grossière de cette grandeur. En effet, on voit sur la figure 17 que le glissement moyen $\overline{\Delta x} / 2$ correspond à

$$
\Delta \gamma_{\mathrm{d}}=\frac{1}{2} \frac{\overline{\Delta x}}{e}=\frac{1}{4} \frac{r}{e} \frac{\tau}{G_{\mathrm{I}}} .
$$

Cela conduit au cisaillement moyen réparti sur l'ensemble du matériau :

$$
\gamma_{\mathrm{d}}=\frac{1}{4} \frac{r}{e} \frac{\tau}{G_{\mathrm{I}}} \frac{\text { volume d'interface }}{\text { volume total }}
$$

Dans le cas d'un matériau polycristallin et en assimilant chaque grain à une sphère de rayon $r$, le rapport $\frac{\text { volume de joint }}{\text { volume total }}$ est de l'ordre de $\frac{3 e}{r+3 e}$.

Il en résulte une intensité de relaxation :

$$
\frac{G_{\mathrm{I}}-G_{\mathrm{R}}}{G_{\mathrm{I}}}=\frac{\gamma_{\mathrm{d}}}{\varepsilon} \neq \frac{3 r f}{4(r+3 e)}
$$

avec

$$
\varepsilon \simeq \frac{\tau}{G_{\mathrm{I}}} \quad \text { (déformation élastique) }
$$

$f$ est un nombre compris entre 0,2 et 0,4 exprimant le fait que toutes les faces du grain ne sont pas sollicitées de la même façon. En outre, dans le cas de solides polycristallins, $r \gg e$ : l'intensité de relaxation est alors comprise entre 0,15 et 0,3 , valeur raisonnablement en accord avec celle donnée par Zener [75] et Kê [76].

On peut donc appliquer ce résultat au cas des solides vitreux : l'intensité de relaxation est alors :

$$
\frac{G_{\mathrm{I}}-G_{\mathrm{R}}}{G_{\mathrm{I}}}=\frac{r}{4 e} \frac{\text { volume d'interface }}{\text { volume total }} \text {. }
$$

Les données expérimentales concernant la relaxation $\beta$ dans les solides vitreux indiquent que cette intensité de relaxation varie généralement entre 0,1 et 1 . Avec les valeurs de $r$ estimées précédemment, on peut en déduire l'ordre de grandeur du rapport :

$\frac{\text { volume d'interface }}{\text { volume total }}$ qui serait compris en $10^{-1}$ et $10^{-3}$

4.3 Modèle De Relaxation $\alpha$. - Au voisinage de la température de transition, le réarrangement coopératif peut se produire dans les régions denses et nous allons montrer qu'il peut en résulter une déformation macroscopique par flux de matière. Nous nous appuierons pour cette analyse sur les théories du fluage diffusion et sur une approche récente de la superplasticité. On trouvera une excellente revue des concepts utilisés ici dans l'ouvrage de Poirier [79]. Dans une approche simple du fluage diffusion, on soumet les domaines de matière comprise entre les interfaces (ou pseudo-grains) $\left(^{6}\right)$, au régime des contrainte représenté sur la figure $19 a$ et équivalent à un cisaillement pur sur des plans à $45^{\circ}$. Il est possible
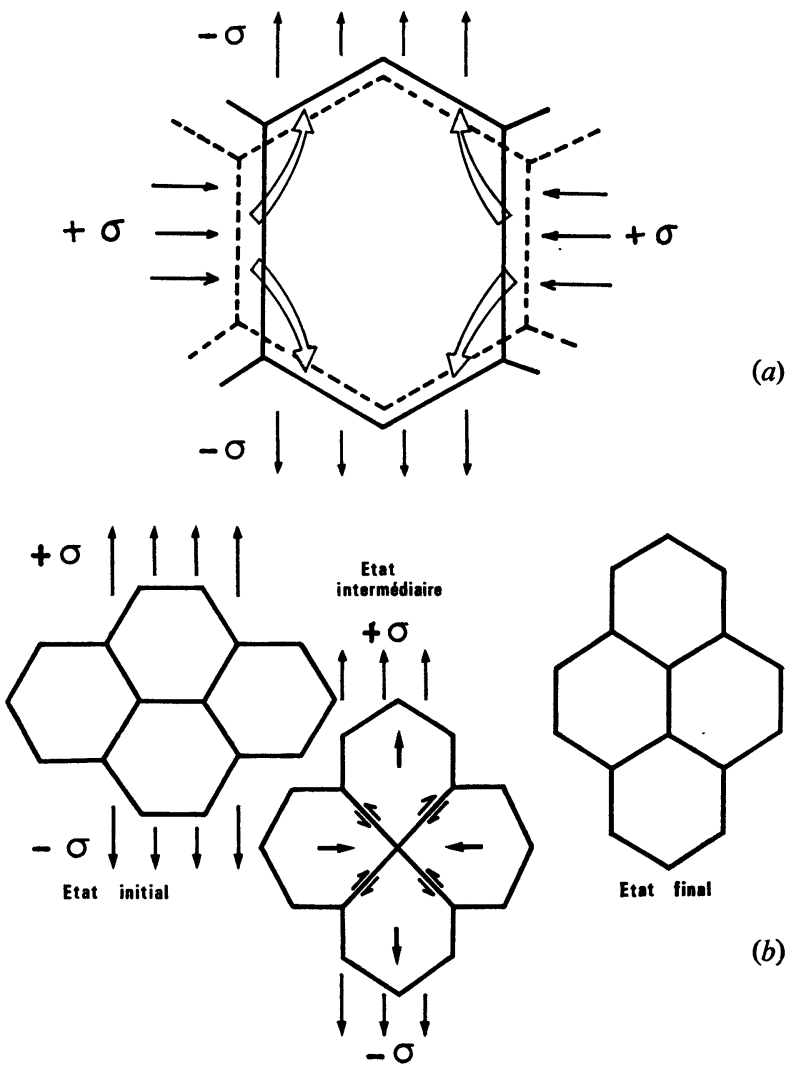

FIG. 19. - Possibilités de déformation dans le pseudo-grain; a) par un mécanisme du type Herring-Nabarro ; b) par un mécanisme proposé par Ashby et Verral dans le cas des matériaux superplastiques [81].

(6) Nous utiliserons le terme de pseudo-grains avec prudence, en rappelant, en particulier, qu'ils correspondent à un arrangement non cristallin des atomes et molécules conformément aux mođèles structuraux généralement admis. 
de déformer ce pseudo-grain par un transport de matière par diffusion, à partir des zones en compression vers les zones en tension c'est-à-dire par fluage Nabarro-Herring. En utilisant le concept de volume libre $v_{\mathrm{f}}$ adopté par Cohen et Turnbull [80] $v_{\mathrm{f}}=\bar{v}-v_{0}$ où $\bar{v}$ est le volume moyen par atome ou molécule et $v_{0}$ le volume de Van der Waals, le volume d'une lacune distribuée ou volume contenant l'équivalent d'un site libre est $\bar{v} \times \bar{v} / v_{\mathrm{f}}$ et la quantité $v_{\mathrm{f}} / \bar{v}^{2}$ donne le nombre de telles lacunes distribuées par unité de volume. Cohen et Turnbull donnent pour $v_{\mathrm{f}}$ l'expression suivante en fonction de la température et de la pression :

$$
v_{\mathrm{f}}=\alpha \bar{v}_{\mathrm{m}}\left(T-T_{0}\right)-\beta \bar{v}_{\mathrm{P}} \Delta P
$$

où $T_{0}$ est la température à laquelle le volume libre disparaît; $\alpha$ et $\bar{v}_{\mathrm{m}}$ représentent respectivement le coefficient d'expansion thermique moyen et le volume moléculaire moyen dans le domaine de température concerné, $\beta$ et $v_{\mathrm{P}}$ sont la compressibilité moyenne et le volume moléculaire moyen pour le domaine de variation de pression choisi.

A température constante $T_{1}$, le nombre de lacunes distribuées par unité de volume dépend donc de la pression par (1) et on peut estimer ce nombre dans les zones comprimées $(+\sigma)$ et tendues $(-\sigma)$.

Dans l'hypothèse simple où $\bar{v}_{\mathrm{m}} \neq \overline{\bar{v}}_{\mathrm{P}} \neq \bar{v}$ :

$$
n(+\sigma)=\frac{\alpha\left(T_{1}-T_{0}\right)-\beta \sigma}{\bar{v}}
$$

et

$$
n(-\sigma)=\frac{\left(T_{1}-T_{0}\right)+\beta \sigma}{\bar{v}}
$$

Il existe donc un gradient de lacunes distribuées entre les zones comprimées et tendues, donc un flux dans le sens de la concentration décroissante en lacunes et un flux de matière en sens inverse donné par la première loi de Fick :

$$
\varphi=-D \operatorname{grad} . n
$$

où $D$ est le coefficient de diffusion proposé par Cohen et Turnbull :

$$
D \# g a^{*} \mathrm{u} \exp \left(-\frac{\bar{v}}{v_{\mathrm{f}}}\right)
$$

où $g$ est un facteur géométrique, $a^{*}$ est de l'ordre du diamètre atomique et $u$ représente la vitesse de déplacement des molécules (donnée par la théorie cinétique des gaz dans l'approche de Cohen et Turnbull).

Il faut bien remarquer que cette diffusion ne se produit pas comme le résultat d'une activation au sens ordinaire du terme mais bien plutôt comme une redistribution des volumes libres à partir de fluctuations dans leur répartition. Cette redistribution entraîne le déplacement des lacunes distribuées et la migration concomittante, en sens inverse, de la matière. A partir de (2) et (3) un calcul classique en fluage diffusion [79] donne la vitesse de déformation relative du pseudo-grain et donc du solide vitreux dans l'hypothèse simple où l'on annule le terme de pression dans le coefficient de diffusion moyen :

$$
\dot{\varepsilon}=\frac{\chi_{1} g a^{*} u \beta}{l^{2}} \exp \left(-\frac{1}{\alpha\left(T-T_{0}\right)}\right) \sigma
$$

$l \sim 2 r$ est le diamètre du pseudo-grain, $\chi_{1}$ est une constante de l'ordre de 10.

En adoptant une contrainte carrée (Fig. 20) pour calculer le frottement intérieur il vient à partir de (5)

$$
\delta=\frac{1}{2} \frac{\Delta W}{W}=\frac{\dot{\varepsilon} M_{\mathrm{I}}}{v \sigma}=\frac{C}{v} \exp \left(-\frac{D}{T-T_{0}}\right)
$$

où $C$ et $D$ sont des constantes et $v$ la fréquence de sollicitation.

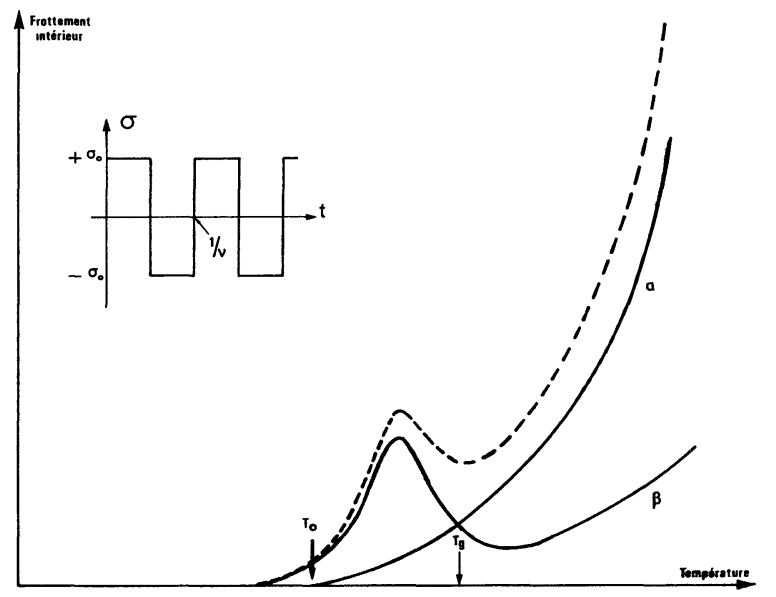

Fig. 20. - Spectres $\delta=f(T)$ théoriques obtenus à partir de l'hypothèse d'une contrafinte carrée et des modèles $\alpha$ et $\beta$

La courbe donnant la composante $\alpha$ du frottement intérieur démarre donc à partir de $T_{0}$ et présente une croissance rapide en fonction de la température ce qui est en bon accord qualitatif avec l'expérience.

En fait, nous devons également tenir compte dans cette montée de frottement intérieur d'une composante associée au mouvement atomique ou moléculaire envisagé dans l'interface ou pseudo-joints (cf. § précédent). Une analyse analogue à la précédente mais où la diffusion se produirait non dans le pseudo-grain mais dans les pseudo-joints conduit, en s'appuyant cette fois sur l'analyse de fluage diffusion proposée par Coble (voir réf. [79]), à l'expression $\left({ }^{7}\right)$ :

$$
\dot{\varepsilon}=\frac{\chi_{2} v_{0} a^{* 2} e \bar{v}}{k T l^{3}} \exp \left(-\frac{A}{k T}\right) \tau
$$

$\chi_{2}$ : constante de l'ordre de $10^{2}$.

(7) Cette expression résulte de l'hypothèse d'une diffusion par sauts thermiquement activés d'atomes (cf. §4.2); il est possible que le déplacement de matière se fasse là aussi, par mouvements coopératifs d'atomes; $\dot{\varepsilon}$ aurait alors une forme plus complexe rappelant (5). 
On voit que ce type de fluage peut participer, pour une part plus ou moins importante, à la montée du fond continu de frottement intérieur qui se produit dans le domaine de relaxation $\beta$.

Dans l'analyse précédente, nous avons supposé qu'au cours du fluage d'un matériau vitreux, la déformation de l'éprouvette résultait directement de la déformation des pseudo-grains.

Bien que cette hypothèse ne soit pas déraisonnable, nous manquons d'éléments expérimentaux pour le confirmer et il n'est pas sans intérêt d'envisager un parallèle avec la superplasticité.

Ce type de comportement qui paraît précisément être celui des verres (allongement considérable de 100 à $2000 \%$ sans apparition de striction) apparaît essentiellement (en dehors des phénomènes de changement de phase) dans les matériaux cristallins à grains très fins déformés à vitesse faible et à température élevée.

Dans l'hypothèse du verre avec interfaces, la relaxation $\alpha$ peut donc être interprétée comme une manifestation analogue à la superplasticité. Dès lors, en reprenant le modèle récent de Ashby et Verrall [81], la déformation d'un verre n'est plus la conséquence de la déformation d'un pseudo-grain unitaire mais d'un changement local de voisin. Dans ces conditions, les pseudo-grains restent équiaxes et le nombre de pseudograins diminue dans la section droite et augmente dans la longueur de l'éprouvette (Fig. 19b).

L'établissement d'une formule donnant $\dot{\varepsilon}$ en fonction de $\sigma$ ferait alors intervenir à la fois la diffusion de matière comme dans les modèles précédents (moyenne pondérée de (5) et (7) et la réaction à l'interface pseudo-grain-pseudo-joint exprimant les échanges de défauts entre les zones de densités différentes). Tenir compte de cette dernière condition demande des hypothèses plus précises sur les processus de diffusion dans les solides non cristallins, mais on peut raisonnablement penser, par analogie avec l'étude citée [81] que cette voie permettrait de faire apparaitre un comportement non newtonien dans les équations d'état $\dot{\varepsilon}=f(\sigma)$. En effet, il apparaît dans les expressions de Ashby et Verrall [81], d'une part une contrainte seuil $\sigma_{0}$ qui conduit à un écoulement caractéristique des fluides de Bingham ; d'autre part, si c'est la réaction d'interface qui contrôle la déformation (en d'autres termes, les pseudo-joints ne sont plus des sources et des puits parfaits pour les lacunes distribuées) on a, en outre, une dépendance en $\sigma^{m}(m \neq 1)$.

La figure 20 résume très schématiquement ce qui précède en montrant comment le frottement intérieur peut résulter de la somme de 2 composantes dues respectivement aux processus $\alpha$ et $\beta$.

5. Discussion et conclusions. - Dans ce qui précède, nous avons postulé la généralité de l'existence des phénomènes de relaxation $\alpha$ et $\beta$ et bâti une interprétation présentant quelque analogie avec le comportement des solides polycristallins.

On peut objecter tout d'abord que les processus $\beta$ loin d'être généraux, sont en fait des cas d'espèce puisqu'ils ne sont pas observés sur tous les spectres de frottement intérieur (ou des caractéristiques diélectriques) en fonction de la température. En réalité, la notion de pic de relaxation $\beta$ implique plus généralement l'existence d'une élasticité retardée directement liée dans notre modèle, à la fixité des joints triples : Or, cette élasticité retardée est souvent signalée dans le cas de matériaux divers : métaux vitreux [45, 82], solides covalents vitreux [78], verres minéraux [8, 27, 83] et organiques [56].

Cette hypothèse et la fixité des joints triples sur laquelle reposent les modèles de Zener [75] et de $\mathrm{Ke}$ [76] mérite sans doute d'être précisée dans le cas des solides vitreux. Bien entendu, dans le cas où sa validité ne serait que partielle, il y aurait une diminution de la force de relaxation du phénomène $\beta$ qui conduirait alors à une montée du frottement intérieur pouvant d'ailleurs se confondre avec celle associée au phénomène $\alpha$.

Certains auteurs ont proposé une description de la double relaxation dans les solides vitreux en termes de modèles rhéologiques : par exemple Koppelmann [72] considère un modèle de Zener, pour rendre compte de la relaxation $\beta$ et, en parallèle avec l'amortisseur, un modèle de Maxwell correspondant à la relaxation $\alpha$.

Dans une approche différente, Wang [84] propose un modèle de Maxwell $(\alpha)$ en série avec une infinité de modèles de Voigt dont le temps de relaxation varie de zéro à l'infini $(\beta)$. Pour notre part, nous présentons dans ce travail une interprétation s'appuyant sur des considérations plus physiques ; néanmoins, il est possible de faire correspondre à cette interprétation et, dans le cas de contraintes faibles, le modèle rhéologique suivant : un modèle de Maxwell avec, en parallèle avec l'amortisseur, un $2^{\mathrm{e}}$ modèle de Maxwell. Un tel modèle rhéologique s'apparente à celui de Koppelmann à ceci près que ce dernier est appliqué à un verre organique macromoléculaire ; or, dans ce cas, $T_{\mathrm{g}}$ est suivi du plateau caoutchoutique et la relaxation $\alpha$ donne lieu à un pic de frottement intérieur du fait de l'existence d'une force de rappel.

Dans la présentation des résultats expérimentaux (partie 2), le comportement non newtonien des solides vitreux vers $T_{\mathrm{g}}$ a été évoqué dans plusieurs cas. Les modèles précédents pourraient nous permettre de comprendre un tel comportement : en effet, la relaxation $\alpha$ peut ne pas être linéaire vis-à-vis de la contrainte pour les raisons suivantes:

- la relation (5) (partie 4) a été obtenue en tenant compte du coefficient de diffusion moyen ; en réalité, ce coefficient de diffusion augmente avec la contrainte mais cette augmentation ne devient sensible que pour des contraintes élevées ;

- conformément au modèle d'Ashby et Verall [81], le passage des pseudo-grains par les positions représentées sur la figure $19 b$ entraîne l'existence d'une contrainte seuil $\sigma_{0}$ dont la valeur est, bien entendu, 
distribuée comme la valeur du diamètre des pseudograins ;

- si la déformation est contrôlée par les réactions à l'interface, $\varepsilon$ varie comme $\sigma^{2}$ quand $\sigma$ est supérieure à $\sigma_{0}$. Cette possibilité est d'autant plus probable que le grain est petit ;

- il n'est pas exclu que le concept de dislocation puisse être encore utilisable dans le cas des solides vitreux [85] : dans ces conditions, aux processus de déformation par simple diffusion, peut s'ajouter un mécanisme de déformation par mouvement de défauts linéaires faisant intervenir ainsi une composante de $\dot{\varepsilon}$ variant comme $\tau^{m}$ avec $m>1$ [79].

Un autre aspect qu'il peut être intéressant de considérer concerne le rôle de la fréquence sur la relaxation $\alpha$. La relation proposée dans la partie 4 pour décrire le frottement intérieur vers $T_{\mathrm{g}}$ indique une dépendance en $v^{-1}$; les études expérimentales portant sur les verres minéraux [26] révèlent une dépendance en $v^{-b}$ avec $b$ de l'ordre de $1 / 3$ à $1 / 2$ lorsqu'un effet d'élasticité retardée accompagne la relaxation $\alpha$, et $b$ tendant vers 1 lorsque le matériau ne présente que la relaxation $\alpha$.

Nous avons postulé l'existence d'interfaces dans les solides vitreux : nous devons nous interroger sur la nature de tels défauts. Des observations en microscopie électronique par transmission effectuées sur des verres silice-oxyde alcalin semblent révéler l'existence de microhétérogénéités dont les dimensions seraient de l'ordre de $100 \AA$ [88]. Dans $\mathrm{SiO}_{2}$ vitreux par contre, aucune sub-microstructure n'est observée mais cela n'exclut que la présence de microcristallites [89]. Moss et Graczyk [61], à partir d'expériences de diffraction d'électrons faites sur du silicium amorphe estiment qu'il existe soit des micro-vides soit des zones de moindre densité. Brodsky et Title [30] constatent que les caractéristiques des spectres de résonance paramagnétique électronique de solides covalents vitreux $(\mathrm{Si}, \mathrm{Ge}, \mathrm{SiC})$ sont comparables à celles des spectres obtenus avec du silicium fraîchement clivé. Ces résultats les conduisent à envisager le modèle des microcristallites séparées par des interfaces riches en atomes possédant des liaisons libres; ils estiment même le nombre de ces atomes qui serait de l'ordre de $10^{20} \mathrm{~cm}^{-3}$.

Ces remarques nous amènent à considérer, dans le cas des solides à liaisons dirigées, une autre possibilité schématisée sur la figure 21: le solide vitreux serait formé de domaines dans lesquels les atomes seraient arrangés conformément au modèle du réseau désordonné ; la juxtaposition de ces domaines se ferait grâce à des pseudo-joints ou interfaces dans lesquels :

- la densité serait moindre, libres,

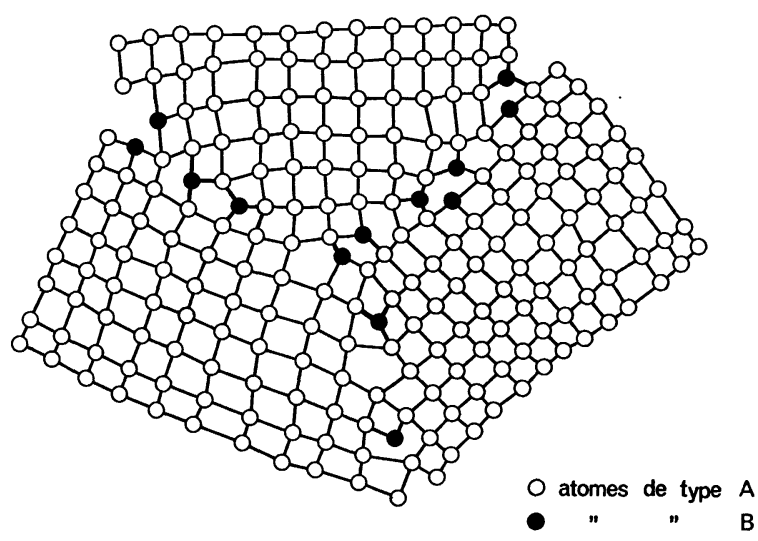

FIG. 21. - Exemple d'interfaces avec point triple dans un solide vitreux à liaisons dirigées. Atomes $\mathrm{A}$ : coordinence normale; atomes $\mathrm{B}:$ atomes avec au moins une liaison libre.

- les liaisons sont plus distordues et les distances entre premiers voisins plus largement distribuées.

Les deux premières caractéristiques sont en accord avec les résultats de Moss et Graczyk [61] d'une part, Brodsky et Title [90] d'autre part.

La $3^{\mathrm{e}}$ caractéristique correspond à la conclusion de Böehr [71] pour qui un verre semi-conducteur serait formé de domaines juxtaposés et entre lesquels existeraient des déformations et des contraintes importantes. Après élaboration du solide vitreux, on ne peut d'ailleurs pas écarter l'hypothèse d'une variation du nombre de liaisons rompues au niveau de l'interface au cours de traitements thermiques.

Dans le cas des métaux vitreux, comme nous l'avons vu au paragraphe 3 , les interfaces pourraient correspondre aux limites de l'arrangement polytétraédrique. Une autre possibilité est liée à la présence de dislocations et à leur aptitude à former des parois.

En résumé, nous avons essayé de montrer que les relaxations $\alpha$ et $\beta$ généralement observées vers la température de transition dans le cas des solides vitreux est caractéristique de l'état structural de ces solides. Pour interpréter ces 2 phénomènes de relaxation, nous avons considéré les modèles structuraux les plus couramment admis et postulé, en outre, l'existence de défauts de type interfaces. Nous avons alors été conduits à associer la relaxation $\beta$ au mouvement thermiquement activé des atomes ou molécules au niveau des interfaces, la relaxation $\alpha$, au contraire, correspondrait au mouvement coopératif de l'ensemble des atomes ou molécules situés entre les interfaces.

L'adaptation des modèles de relaxation existant dans le domaine des matériaux polycristallins, au cas des solides vitreux nous a permis une interprétation au moins qualitative des résultats expérimentaux et une évaluation de la distance moyenne entre interfaces qui serait de l'ordre de $100 \AA$. 


\section{Bibliographie}

[1] Roy, R., J. Non Crystall. Solids. 3 (1970) 33

[2] Scholze, H., «le verre » (Inst. du verre) 1969.

[3] Johari, G. P., à paraître (1976), J. Chem. Phys. 58 (1973) 1766.

[4] Turnbull, D. et PoKL, D. E., J. Non Crystall Solids. 8-10 (1972) 19.

[5] GuYe, C. E. et Vasileferf, S., Arch. Sci. Nat. 37 (1914) 214.

[6] KIRBY, P. L., J. Soc. Glass. Tech., Part. I (1953), 7T, Part. II (1954) 383 T, Part. III (1954), 548 T ; Trans. Farad. Soc., 52 (1956) 131.

[7] Rindone, G. E., Min. Ind. 31 (1961) 1.

[8] Hopkins, I. L. et KurkJian, C. R., Phys. Ac. II B, ed. Mason W. E. (Acad. Press), 1965, 91.

[9] Douglas, R. W., Relax. Proc. in glasses, Ed. Day D. E. (North Hol. Pub. Co) 1974, 1 ; «Amorph. Mat.», Ed. Douglas R. W. et Ellis (Wiley Int.) 1972, 3.

[10] Aziber T, C., thèse, Lyon (1971).

[11] DAY, E. D., « Amorph. Mat. », Ed. Douglas R. W. et Ellis B. (Wiley Int.), 1972, 39.

[12] Angell, C. A. et Wong, J., J. Chem. Phys. 53 (1970) 2053.

[13] Angell, C. A. et Melphrey, D. B., J. Phys. Chem. 75 (1971) 2306.

[14] Jackle, J., Piche, L., Arnold, W. et Munklinger, S., $J$. Non Crystall. Solids 20 (1976) 365.

[15] Doussineau, P., Revue Phys. Appl. 12 (1977) 809.

[16] TAYLOR, T. D. et Rindone, G. E., " Relax. Proc. ni glasses » Ed. Day D. E. (North Hol. Pub. Co), 1974, 157.

[17] Hoffman, L. C. et WeYL, W. A., The Glass Indust. 38, 1957) 81.

[18] BRÜCKneR, R., J. Non Crystall. Solids 5 (1970-71), 123.

[19] Mohyuddin, I. et Douglas, R. W., Phys. Chem. glass 1 (1960) 71.

[20] Day, D. E. et Rindone, G. E., J. Am. Cer. Soc. 45 (1962) 496.

[21] DAY, D. E., Wiss, Zeitschr. N 2 (1974) 293.

[22] Day, D. E. et Stevels, J. M., Relax. Proc. in glasses, Ed. DAY D. E. (North Hol. Pub. Co) 1974, 165.

[23] Day, D. E. et Rindone, G. E., J. Am. Cer. Soc. 44 (1961) 161.

[24] Weber, N. et Goldstein, M., J. Chem. Phys. 41 (1964) 2898.

[25] VöLkt, J., Ber. Bunsen Gesel. Phys. Chem. 76 (1972) 797.

[26] Douglas, R. W., Duke, P. J. et Mazurin, O. V., Phys. Chem. Glass 9 (1968) 169.

[27] Argon, A. S., J. Appl. Phys. 39 (1968) 4080.

[28] Shelby, J. E. et DAY, D. E., J. Am. Cer. Soc. 21 (1969) 169.

[29] Shelby, J. E., J. Am. Cer. Soc. 53 (1970) 226.

[30] McVaY, C. L. et DaY, D. E., J. Am. Cer. Soc. 53 (1970) 508.

[31] Van Ass, H. M. J. M., Thèse, Eindhoven (1975).

[32] Day, D. E. et Stevels, J. M., J. Non Crystall. Solids 11 (1973) 459.

[33] Verstegen, E. H. et Day, D. E., Relax. Pric. in Glasses, Ed. Day D. E. (North. Hol. Pub. Co) 1974, 142.

[34] Van Gemert, W. J. T., Ban Ass H. M.. J. M. et Stevels, J. M., J. Non Crystall. Solids 16 (1974) 281.

[35] Phalippou, J., Masson, S., Boyer, A. et Zarzycki, J., "Relax. Proc. in Glasses », Ed. Day D. E. (North Hol Pub. Co) 1974178.

[36] DAy, D. E., J. Am. Cer. Soc. 57 (1974) 530.

[37] Graham, P. W. L. et Rindone, G. E., Phys. Chem. Glass. 8 (1967) 160.

[38] Day, D. E. et Steinkamp, W. E., J. Am. Ceram. Soc. 52 (1969) 571.

[39] Ismail, A., Abdel-Latif, A. et Day, D. E., J. Am. Ceram. Soc. 55 (1972) 279.

[40] Ismail, A., Abdel-Latif, A. et Day, D. E., J. Am. Ceram. Soc. 55 (1972) 254.

[41] Gilman, J. J., J. Appl. Phys. 46 (1975) 1625.

[42] Chen, H. S. et Turnbull, D., Acta Metall. 17 (1969) 1021.

[43] Chen, H. S., Leamy, H. J. et Barmatz, M., J. Non Crystall. Solids 5 (1971) 444.
[44] Eisenberg, A. et Reich, S., Amorp. Mat. Ed. Douglas R. W. et Ellis B. (Wiley Int.), 1972, 39.

[45] Chen, H. S. et Goldstein, M., J. Appl. Phys. 43 (1972) 1642.

[46] Imaoka, M. et SaKamura, H., Glass Techn. 15 (1974) 105.

[47] Graham, L. J. et Chang, R., J. Appl. Phys. 36 (1965) 2983.

[48] Amrhein, E. M., Day, D. E. et Kreidl, N. J., J. Non Crystall. Solids 16 (1974) 46.

[49] Cukierman, M. et Uhlmann, D. R., J. Non Crystall. Solids 12 (1973) 199.

[50] Pineri, M. et Moser, P., Col. Int. Lausanne (1970).

[51] Ferry, J. D., Visoel. Prop. Polym., (Wiley) 1970.

[52] McCrum, N. G., Read, B. E. et Williams, G., Anel and Dielect. effects in Polym. Sol (Wiley) 1967.

[53] Heijboer, J., Thèse, Delft (1972).

[54] Heibjoer, J., Att. $2^{\mathrm{e}}$ conv. soc. Ital. Réol (Siene) 10 (1973) 307.

[55] Wert, C. et Marx, J., Acta Met. 1 (1953) 113.

[56] Harrison, G., " the dynam Prop. of supercooled liq.", (Acad. Press) 1976.

[57] Barlow, A. J., Day, M., Harrisson, G., Lamb, J. et Subramanian, S., Proc. Roy. Soc. A 309 (1969), 597.

[58] Illers, K. H., Rheol. Acta 4 (1964), 185.

[59] Faucher, J. A. et Koleske, J. V., Phys. Chem. Glass. 7 (1966), 202.

[60] MAUCET, G., Rap. CEA-CENG-SBT (1976)

[61] Moss, S. C. et CrazcyK, J. P., Phys. Rev. Lett. 23 (1969) 1167.

[62] Grigorovici, R. et Manaila, R., J. Non Crystall. Solids 1 (1969) 371.

[63] Goldstein, M., J. Physique Colloq. 36 (1975) C 2-33.

[64] Zachariasen, W. H., J. Am. Chem. Soc. LIV (1932) 3841.

[65] Polk, D. E., J. Non Crystall. Solids 5 (1971) 365.

[66] Popescu, M., Phys. State Solids 35 (1976) 355.

[67] Johari, G. P. et Goldstein, M., J. Chem. Phys. 53 (1970) $2372 ; 55$ (1971) 4245.

[68] Goldstein, M., " Amorp. Mat. », Ed. Douglas R. et Ellis B. (Wiley), 1972, 23. Farad. Symp. Chem. Soc. 6 (1972) 7.

[69] Williams, G. et WatTs, D. C., Trans. Farad. Soc. 67 (1971) 1971.

[70] Brodsky, M. M., Title, R. S., Weiser, K. et Petrt, G. D., Phys. Rev. B 1 (1970) 2632.

[71] Bö̈r, K. W., J. Non Cryst. Solids 8-10 (1972) 586.

[72] Koppelmann, J., « Phys. Non Crystall Solids », Ed. Prins J. A., (North Hol. Pub. Co) 1965, 255.

[73] Nowick, A. S. et BerRy, B. S., « Anel Relax. in Cryst. Sol. » (Acad. Press) 1972292.

[74] Rouby, D. et Gobin, P. F., Phil. Mag. 29 (1974) 983.

[75] Zener, C., Phys. Rev. 60 (1941) 906.

[76] Ke, T. S., Phys. Rev. 71 (1947) 533.

[77] Ke, T. S., J. Appl. Phys. 20 (1949) 274.

[78] Etienne, S. et Perez, J., Revue Phys. Appl. 12 (1977) 837.

[79] PoIrIER, J. P., « Plast. des Sol. Crist. », Eyrolles (1976).

[80] Cohen, M. H. et Turnbull, D., J. Chem. Phys. 31 (1959) 1164.

[81] Ashby, M. F. et Verrall, R. A., Acta Met. 21 (1973) 149.

[82] Chen, H. S. et Wang, T. T., J. Appl. Phys. 13 (1970) 5338.

[83] LeVengood, W. C. et Vong, T. S., J. Opt. Soc. Amer. 49 (1959) 61.

[84] Wang, F. F. Y., J. Appl. Phys. 6 (1962) 2065.

[85] Gilman, J. J., « Disl. Dynam.» Ed. Rosenfield, Hahn, Bement, Jaffee (Mc Graw Hill), 1967, 3.

[86] Polk, D. E., Acta Met. 20 (1972) 485.

[87] Pampillo, C. A. et Chen, H. S. Mat. Sci. Eng. 13 (1974) 181.

[88] Seward, T. P., UhlmanN, D. R., Turnbull, D. et PierCe, G. R., J. Am. Ceram. Soc. 50 (1967) 25.

[89] Seward, T. P. et Uhlmann, D. R., Amorp. Mat. ed. Douglas P. W. et Ellis B. (Wiley) 1970, 121.

[90] Brodsky, M. H. et Tirte, R. S., Phys. Rev. Lett. 23 (1969) 581. 